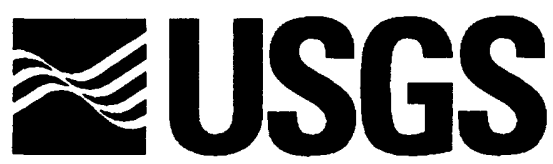

science for a changing world

Prepared in cooperation with the

ARKANSAS SOIL AND WATER CONSERVATION COMMISSION, the ARKANSAS GEOLOGICAL COMMISSION, and the LOUISIANA DEPARTMENT OF TRANSPORTATION AND DEVELOPMENT

POTENTIOMETRIC SURFACE OF THE SPARTA AQUIFER IN EASTERN AND SOUTH-CENTRAL ARKANSAS AND NORTH-CENTRAL LOUISIANA, AND THE MEMPHIS AQUIFER IN EAST-CENTRAL ARKANSAS, OCTOBER 1996-JULY 1997

Water-Resources Investigations Report 97-4282

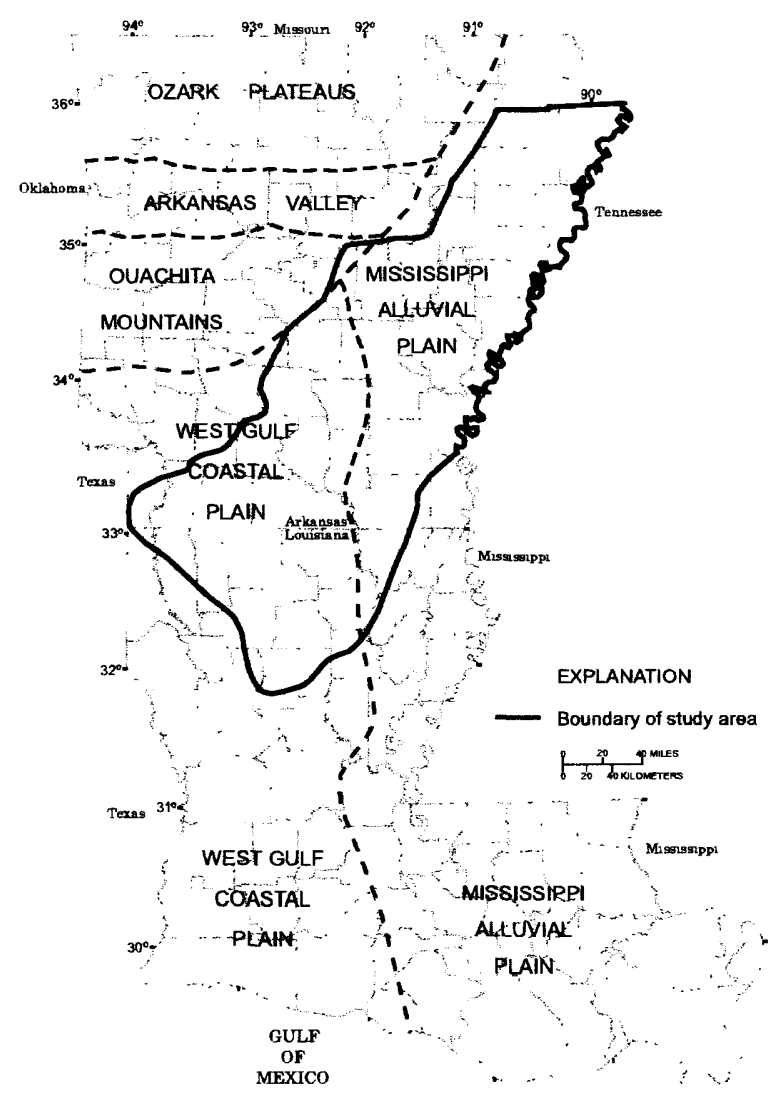

U.S. Department of the Interior

U.S. Geological Survey 
POTENTIOMETRIC SURFACE OF THE SPARTA AQUIFER IN EASTERN AND SOUTH-CENTRAL ARKANSAS AND NORTH-CENTRAL LOUISIANA, AND THE MEMPHIS AQUIFER IN EAST-CENTRAL ARKANSAS, OCTOBER 1996-JULY 1997

By Robert L. Joseph

U.S. GEOLOGICAL SURVEY

Water-Resources Investigations Report 97-4282

Prepared in cooperation with the ARKANSAS SOIL AND WATER CONSERVATION COMMISSION, the ARKANSAS GEOLOGICAL COMMISSION, and the LOUISIANA DEPARTMENT OF TRANSPORTATION AND DEVELOPMENT 


\section{U.S. DEPARTMENT OF THE INTERIOR \\ BRUCE BABBITT, Secretary}

\section{U.S. GEOLOGICAL SURVEY}

Mark Schaefer, Acting Director

For additional information write to:

\section{District Chief}

U.S. Geological Survey, WRD

401 Hardin Road

Little Rock, Arkansas 72211
Copies of this report can be purchased from:

U.S. Geological Survey

Branch of Information Services

Box 25286

Denver Federal Center

Denver, Colorado 80225 


\section{CONTENTS}

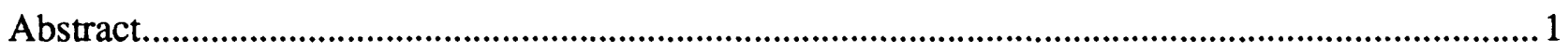

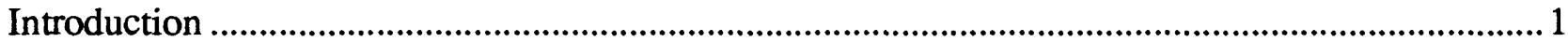

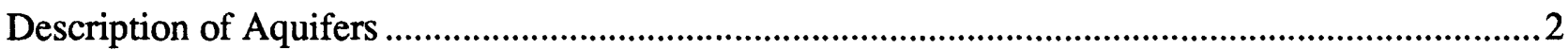

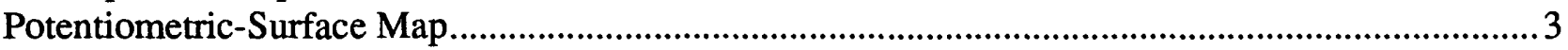

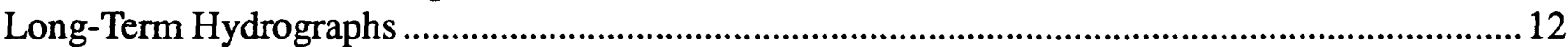

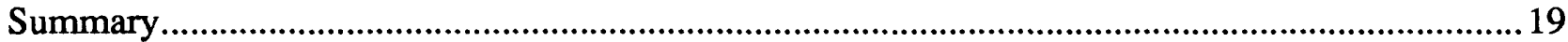

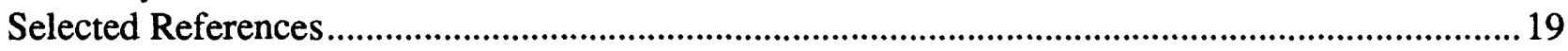

\section{PLATES}

Plate 1. Map showing potentiometric surface of the Sparta and Memphis aquifers.......... In pocket

\section{ILLUSTRATIONS}

Figure 1. Map showing location of study area ...............................................................................

2. Water-level hydrographs for selected wells completed in the Sparta and Memphis aquifers

\section{TABLES}

Table 1. Information pertaining to measured wells completed in the Sparta and Memphis aquifers in Arkansas...

2. Information pertaining to measured wells completed in the Sparta aquifer in Louisiana.11 


\title{
POTENTIOMETRIC SURFACE OF THE SPARTA AQUIFER IN EASTERN AND SOUTH-CENTRAL ARKANSAS AND NORTH-CENTRAL LOUISIANA, AND THE MEMPHIS AQUIFER IN EAST-CENTRAL ARKANSAS, OCTOBER 1996-JULY 1997
}

\author{
by Robert L. Joseph
}

\section{ABSTRACT}

During the 1997 water year, the water level in the Sparta and Memphis aquifers was measured in about 274 wells in Arkansas, and the water level in the Sparta aquifer was measured in about 55 wells in Louisiana. The potentiometric surface data reveal spatial trends across the study area. In Arkansas, the altitude of the potentiometric surface ranged from 199 feet below sea level in Union County to 307 feet above sea level in Saline County. In Louisiana, the altitude of the potentiometric surface ranged from 224 feet below sea level in Ouachita Parish to 230 feet above sea level in Bienville Parish.

The general direction of ground-water flow in the Sparta and Memphis aquifers is from the west to the southeast. The regional direction of ground-water flow in Arkansas is from the north and west to the south and east, away from the recharge zone in the outcrop and subcrop area, except near areas affected by intense ground-water withdrawals; such areas are manifested by large cones of depression centered in Columbia, Jefferson, and Union Counties. The regional groundwater flow in the Sparta aquifer in north-central Louisiana generally is downdip in an easterly direction from the recharge zone in the outcrop and subcrop area in the west toward the Mississippi Alluvial Plain. The potentiometric surface of the Sparta aquifer in Arkansas and Louisiana exhibits cones of depressions descending below sea level. Comparison of potentiometric surface maps through time shows that the cones of depression in Columbia and Union Counties are coalesc- ing at or near the Columbia and Union County line. However, the general direction of groundwater movement indicates that heavy pumpage locally has altered or reversed the natural direction of flow in some areas. Flow in these areas is toward the cones of depression at the center of pumping.

Hydrographs from wells in the Sparta and Memphis aquifers reveal that water levels have declined more than 2.0 feet per year in some wells. Long-term hydrographs of eight wells in Arkansas, during the period 1972-1997, reveal waterlevel declines ranging from less than 0.8 foot per year in Phillips County to more than 2.0 feet per year in Union County. Long-term hydrographs of two wells in Louisiana, during the period 19721997, reveal water-level declines were more than 2.0 feet per year in Lincoln and Ouachita Parishes.

\section{INTRODUCTION}

The Sparta and Memphis aquifers are major sources of water for eastern and south-central Arkansas and north-central Louisiana, where in 1995 more than 355 million gallons per day $(\mathrm{Mgal} / \mathrm{d})$ of water was withdrawn. In Arkansas, major withdrawals are made from the aquifers for industrial and public supply, with lesser but locally significant withdrawals for agricultural uses. An estimated $284 \mathrm{Mgal} / \mathrm{d}$ of water was withdrawn from the Sparta and Memphis aquifers in 1995, an increase of about $61 \mathrm{Mgal} / \mathrm{d}$ from 1990. The two aquifers are the second most productive source of ground water in Arkansas (Holland, 1993). In Louisiana, major withdrawals are made from the aquifer for industrial and public supply, with lesser withdrawals 
for other uses. An estimated $71 \mathrm{Mgal} / \mathrm{d}$ of water was withdrawn from the Sparta aquifer in 1995, an increase of about $7 \mathrm{Mgal} / \mathrm{d}$ from 1990.

The study area (fig. 1, plate 1) includes most of the Coastal Plain physiographic province in Arkansas and Louisiana. In Arkansas, the area is bounded on the north by the Missouri State line, and on the east by the Tennessee and Mississippi State lines. The western boundary is defined as the western extent of the outcrop and subcrop (Hosman, 1982) of the Sparta Sand and the Memphis Sand. In Louisiana, the area is bounded on the south and east by the approximate downdiplimit of freshwater (Payne, 1968) and the western boundary is defined by the western limit of the Sparta aquifer.

The U.S. Geological Survey in cooperation with the Arkansas Soil and Water Conservation Commission, the Arkansas Geological Commission, and the Louisiana Department of Transportation and Development has monitored water levels in the Sparta and Memphis aquifers since the 1920's. During the 1997 water year (October 1996 through September 1997), about 274 water-level measurements in Arkansas and about 55 water-level measurements in Louisiana were made in wells completed in these aquifers. The purpose of these measurements was to provide information describing the potentiometric surface of the Sparta and Memphis aquifers. This report presents results as a map and as updated water-level hydrographs.

The author acknowledges the contribution of data from the Louisiana District, U.S. Geological Survey, and the technical assistance of their staff, particularly Jeffrey A. Brantley, in the publication of this report.

\section{DESCRIPTION OF AQUIFERS}

The Sparta Sand and Memphis Sand of Eocene age are part of the Claiborne Group and mainly consist of fine- to medium-grain sand. In the central and southern parts of the study area, the Sparta Sand is underlain by the marine clay of the Cane River Formation, which serves as a lower confining unit. In the northern part of the study area (north of about 35 degrees latitude), the Cane River Formation is predominantly composed of sand (Hosman and others, 1968), and the Memphis Sand is thicker and more homogeneous. In this northem area, the Claiborne Group is not subdivided into the Sparta Sand, Cane River Formation, and Carrizo Sand, but the equivalent section is a single formation known

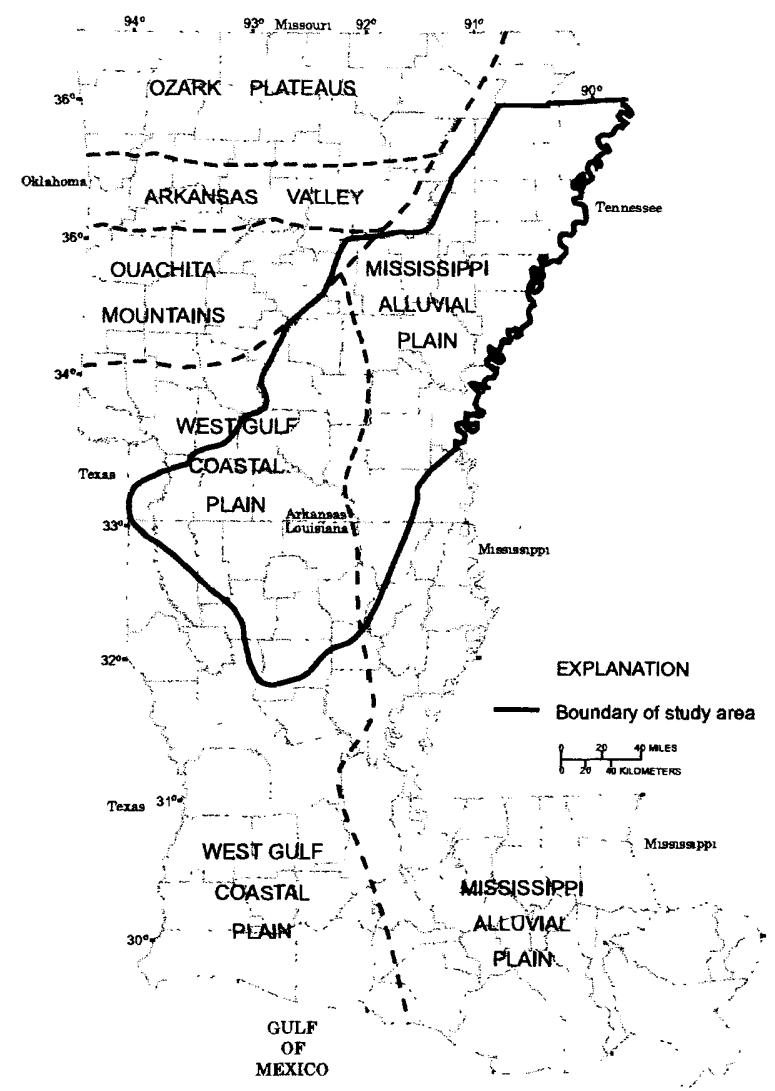

Figure 1. Location of study area (physiographic boundaries from Fenneman, 1938). 
as the Memphis Sand. The Memphis Sand is underlain by a thick layer of clay that is part of the Wilcox Group. Some silt, clay, and lignite occur in the upper portion of the Sparta and Memphis Sands. The Sparta Sand in north-central Louisiana consists of very fine- to medium-grain sand, silty clay, lignite, and lesser amounts of clay. The Sparta Sand is composed of a sequence of alternating sand and clay beds between the massive clays of the overlying Cook Mountain and the underlying Cane River confining units. Sands in the Sparta Sand were deposited by shifting streams on a deltaic-fluvial flood plain (Payne, 1968). These sands are mostly interconnected, but separately identifiable sands can be traced for only short distances (Snider and others, 1972). The Cook Mountain Formation overlies the Sparta Sand and Memphis Sand and it serves as an upper confining unit. The permeable units of the Sparta Sand and the Memphis Sand compose the respective aquifers. Water levels in the Sparta aquifer generally correlate with those in the Memphis aquifer; therefore, the water-bearing formations are considered to be one hydrologic unit (Stanton, 1997).

In Arkansas, the Sparta Sand generally thickens and begins to contain saltwater as depth of occurrence increases to the southeast. The Sparta Sand is 50 to 200 feet (ft) thick within the recharge zone (along its western limit) and thickens easterly to nearly $900 \mathrm{ft}$. The Sparta Sand contains freshwater throughout most of its extent in Arkansas. However, saltwater is present in the extreme southeastern part of the State in parts of Ashley, Chicot, and Union Counties.

In Louisiana, the Sparta Sand generally dips to the east and southeast. From the Sabine uplift (plate 1), the dip in northwestern Louisiana is northeasterly. The Sparta Sand is $50-300 \mathrm{ft}$ thick within the recharge zone and thickens easterly to nearly $700 \mathrm{ft}$ near the downdip limit of freshwater. The approximate downdip limit of freshwater is shown on plate 1. Below the downdip limit of freshwater, all sands in the Sparta aquifer contain saltwater; updip from the freshwater limit, sands in the upper part of the aquifer contain freshwater but some sands in the lower part of the aquifer contain saltwater. A more detailed description of the Sparta and Memphis aquifers is given in Edds and Fitzpatrick, 1989; Hosman and others, 1968; Payne, 1968; Petersen and others, 1985; and Ryals, 1980.

\section{POTENTIOMETRIC-SURFACE MAP}

The potentiometric-surface map shows the altitude to which water would have risen in tightly cased wells screened in the aquifers (plate 1). The map is based upon water-level data collected in about 329 wells in the Sparta and Memphis aquifers from October 1996 to July 1997 in eastern and south-central Arkansas and north-central Louisiana. The surface is mapped by determining the altitude of the water levels measured in the wells and is represented on the map by contours that connect points of equal value. The general direction of ground-water flow in the Sparta and Memphis aquifers is perpendicular to the contours in the direction of downward hydraulic gradient.

The natural direction of flow, which is generally eastward from the recharge zone and then southward, is altered in areas of heavy pumpage. The regional direction of ground-water flow in Arkansas is generally to the south to southwest in the northern half of the Arkansas part of the study area and to the east and south in the southern half of Arkansas, away from the recharge zone of the outcrop and subcrop area except where affected by intense ground-water withdrawals. The natural ground-water flow in the Sparta aquifer in northern Louisiana generally is in an easterly direction from the recharge zone of the outcrop and subcrop area in the west toward the Mississippi Alluvial Plain (Ryals, 1980). The highest water-level altitude measured in Arkansas was $307 \mathrm{ft}$ above sea level, located in Saline County near the recharge zone of the outcrop and subcrop; the lowest water level was $199 \mathrm{ft}$ below sea level in Union County (table 1). The highest waterlevel altitude measured in Louisiana was $230 \mathrm{ft}$ above sea level, located in Bienville Parish, near the recharge zone of the outcrop and subcrop area; the lowest water level was $224 \mathrm{ft}$ below sea level in Ouachita Parish (table 2). However, the potentiometric surface indicates that heavy pumpage has altered or reversed the natural direction of flow in some areas. Flow in these areas is toward the cones of depression at the center of pumping. Four large cones of depression are observed, centered in Columbia, Jefferson, and Union Counties in Arkansas and Ouachita Parish in Louisiana as a result of large withdrawals of water for industrial and public supplies. In Arkansas, the potentiometric surface of the Sparta and Memphis aquifers exhibits cones of depression descending below sea level in the central and southwestern parts of the State. The cone of depression centered in Jefferson County has an elliptical shape because of withdrawals for irrigation in the 
Table 1. Information pertaining to measured wells completed in the Sparta and Memphis aquifers in Arkansas

\begin{tabular}{|c|c|c|c|c|c|c|c|}
\hline $\begin{array}{r}\text { Latitude } \\
\text { (degrees) }\end{array}$ & $\begin{array}{c}\text { Longitude } \\
\text { (degrees) }\end{array}$ & Local well number & $\begin{array}{l}\text { Water level } \\
\text { altitude } \\
\text { (feet above } \\
\text { sea level) }\end{array}$ & $\begin{array}{c}\text { Depth to } \\
\text { water } \\
\text { (feet below } \\
\text { land-surface } \\
\text { datum) }\end{array}$ & $\begin{array}{l}\text { Land-surface } \\
\text { datum altitude } \\
\text { (feet above } \\
\text { sea level) }\end{array}$ & $\begin{array}{c}\text { Date of } \\
\text { measure- } \\
\text { ment }\end{array}$ & Aquifer \\
\hline \multicolumn{8}{|c|}{ Arkansas County } \\
\hline 340030 & 0911447 & 08S02W09BCC1 & 79 & 95.31 & 174 & $06-06-97$ & Sparta \\
\hline 340116 & 0911131 & 08S02W01CBA1 & 92 & 73.26 & 165 & $03-25-97$ & Sparta \\
\hline 340124 & 0912039 & 08S03W04DBCl & 84 & 91.87 & 176 & $03-25-97$ & Sparta \\
\hline 340135 & 0912152 & 08S03W05ABB 1 & 72 & 103.21 & 175 & $03-24-97$ & Sparta \\
\hline 340340 & 0911410 & 07S02W28ABA1 & 94 & 86.78 & 181 & $03-25-97$ & Sparta \\
\hline 340711 & 0912248 & 07S03W06ABC1 & 71 & 114.14 & 185 & $03-25-97$ & Sparta \\
\hline 340858 & 0912008 & 06S03W27BAA1 & 74 & 106.98 & 181 & $06-06-97$ & Sparta \\
\hline 341023 & 0911451 & 06S02W17ADA1 & 83 & 105.42 & 188 & $06-06-97$ & Sparta \\
\hline 341228 & 0911622 & 06S02W06ABB 1 & 74 & 106.76 & 181 & $03-26-97$ & Sparta \\
\hline 341247 & 0912946 & 05S05W36DAA1 & 53 & 126.99 & 180 & $03-27-97$ & Sparta \\
\hline 341358 & 0912435 & 05S04W26ACA1 & 65 & 122.97 & 188 & $03-26-97$ & Sparta \\
\hline 341550 & 0910742 & 05S01W17BAA1 & 77 & 99.27 & 176 & $06-05-97$ & Sparta \\
\hline 341734 & 0912006 & 05S03W04ADB 1 & 63 & 123.98 & 187 & $03-26-97$ & Sparta \\
\hline 341752 & 0913004 & 04S05W36DCC1 & 48 & 148.09 & 196 & $03-26-97$ & Sparta \\
\hline 341819 & 0913139 & 04S05W34DAA1 & 38 & 154.09 & 192 & $06-05-97$ & Sparta \\
\hline 341819 & 0913448 & 04S05W31DDA1 & 36 & 148.19 & 184 & $06-10-97$ & Sparta \\
\hline 341929 & 0910739 & 04S01W28BAA1 & 94 & 95.66 & 190 & $03-26-97$ & Sparta \\
\hline 342004 & 0912514 & 04S04W22DAA1 & 57 & 138.08 & 195 & $03-26-97$ & Sparta \\
\hline 342005 & 0912926 & 04S04W19CBB1 & 48 & 147.48 & 195 & $03-26-97$ & Sparta \\
\hline 342123 & 0911331 & 04S02W09DDCl & 115 & 59.65 & 175 & $06-05-97$ & Sparta \\
\hline 342132 & 0913133 & 04S05W15AAA1 & 39 & 162.24 & 201 & $06-05-97$ & Sparta \\
\hline 342155 & 0912503 & 04S04W $11 \mathrm{BCC}$ & 54 & 143.58 & 198 & $03-26-97$ & Sparta \\
\hline 342226 & 0910758 & 04S01W04CBD1 & 93 & 103.25 & 196 & $06-05-97$ & Sparta \\
\hline 342416 & 0912437 & 03S04W26CDA1 & 57 & 145.52 & 203 & $03-26-97$ & Sparta \\
\hline 342416 & 0912645 & 03S04W33BAA1 & 60 & 141.34 & 201 & $03-26-97$ & Sparta \\
\hline 342447 & 0913238 & 03S05W28DAB1 & 37 & 167.37 & 204 & $06-05-97$ & Sparta \\
\hline 342515 & 0914210 & 03S06W30BBD1 & 47 & 143.77 & 191 & $03-27-97$ & Sparta \\
\hline 342554 & 0913925 & 03S06W21 ACCl & 54 & 140.92 & 195 & $03-27-97$ & Sparta \\
\hline 342632 & 0913005 & 03S05W 13BDCl & 39 & 170.59 & 210 & $03-26-97$ & Sparta \\
\hline 342632 & 0913227 & 03S05W15CBB1 & 44 & 162.33 & 206 & $03-26-97$ & Sparta \\
\hline 342633 & 0913523 & 03S05W 18CAB1 & 46 & 149.82 & 196 & $03-27-97$ & Sparta \\
\hline 342747 & 0912457 & 03S04W02CCB1 & 61 & 140.97 & 202 & $03-26-97$ & Sparta \\
\hline 342839 & 0913032 & 03S05W02AAB1 & 65 & 144.58 & 210 & $03-26-97$ & Sparta \\
\hline 342924 & 0912700 & 02S04W33BBB1 & 58 & 147.28 & 205 & $03-26-97$ & Sparta \\
\hline 342930 & 0913034 & $02505 W 35 \mathrm{AAB} 1$ & 35 & 181.03 & 216 & $06-04-97$ & Sparta \\
\hline 343028 & 0913230 & $02505 W 27 \mathrm{BBB} 1$ & 49 & 166.54 & 216 & $03-27-97$ & Sparta \\
\hline 343044 & 0912349 & 02S04W23DAA1 & 84 & 124.09 & 208 & $03-27-97$ & Sparta \\
\hline 343143 & 0913318 & $02505 W 16 \mathrm{CBCl}$ & 21 & 191.86 & 213 & $06-04-97$ & Sparta \\
\hline 343312 & 0912849 & 02S04W06CDB 1 & 64 & 148.34 & 212 & $03-27-97$ & Sparta \\
\hline \multicolumn{8}{|c|}{ Ashiey County } \\
\hline 332117 & 0915103 & 15S07W32CDD1 & 37 & 153.28 & 190 & $03-20-97$ & Sparta \\
\hline
\end{tabular}


Table 1. Information pertaining to measured wells completed in the Sparta and Memphis aquifers in Arkansas--Continued

\begin{tabular}{|c|c|c|c|c|c|c|c|}
\hline $\begin{array}{l}\text { Latitude } \\
\text { (degrees) }\end{array}$ & $\begin{array}{c}\text { Longitude } \\
\text { (degrees) }\end{array}$ & Local well number & $\begin{array}{c}\text { Water level } \\
\text { altitude } \\
\text { (feet above } \\
\text { sea level) }\end{array}$ & $\begin{array}{l}\text { Depth to } \\
\text { water } \\
\text { (feet below } \\
\text { land-surface } \\
\text { datum) }\end{array}$ & $\begin{array}{l}\text { Land-surface } \\
\text { datum altitude } \\
\text { (feet above } \\
\text { sea level) }\end{array}$ & $\begin{array}{l}\text { Date of } \\
\text { measure- } \\
\text { ment }\end{array}$ & Aquifer \\
\hline \multicolumn{8}{|c|}{ Bradley County } \\
\hline 331836 & 0922052 & $16 S 12 W 21 C A A 1$ & 28 & 71.56 & 100 & $04-05-97$ & Sparta \\
\hline 333453 & 0921607 & 13S11W17BCD1 & 54 & 195.69 & 250 & $04-03-97$ & Sparta \\
\hline 333647 & 0920437 & 13S09W06ACB2 & 15 & 186.36 & 201 & $04-02-97$ & Sparta \\
\hline 333649 & 0920406 & 13S09W06BDC1 & 42 & 170.00 & 212 & $04-02-97$ & Sparta \\
\hline 334103 & 0920752 & 12S10W10BCA1 & 45 & 117.61 & 163 & $05-22-97$ & Sparta \\
\hline \multicolumn{8}{|c|}{ Calhoun County } \\
\hline 332811 & 0922722 & 14S13W29DAC1 & 134 & 13.83 & 148 & $04-03-97$ & Sparta \\
\hline 333040 & 0922403 & 14S13W12CCB1 & 35 & 170.22 & 205 & $04-02-97$ & Sparta \\
\hline 333055 & 0923910 & 14S15W16BAA1 & 71 & 75.39 & 146 & $04-02-97$ & Sparta \\
\hline 333226 & 0922741 & 13S13W32CDA1 & 35 & 172.81 & 208 & $03-19-97$ & Sparta \\
\hline 333233 & 0922224 & 13S12W31DAA1 & 133 & 67.26 & 200 & $04-03-97$ & Sparta \\
\hline 333252 & 0923616 & 13S15W36CBD1 & 105 & 53.24 & 158 & $04-02-97$ & Sparta \\
\hline 334630 & 0922927 & $11 S 14 W 12 C A C 3$ & 172 & 141.43 & 313 & $03-17-97$ & Sparta \\
\hline 332407 & 0922810 & $15 S 13 W 20 B D C 1$ & 26 & 82.49 & 108 & $11-06-96$ & Sparta \\
\hline \multicolumn{8}{|c|}{ Chicot County } \\
\hline 333301 & 0911419 & 13S01W19CAA1 & 67 & 64.43 & 131 & $03-24-97$ & Sparta \\
\hline \multicolumn{8}{|c|}{ Cleveland County } \\
\hline 334543 & 0921422 & 11S11W16AAB1 & 91 & 212.09 & 303 & $04-09-97$ & Sparta \\
\hline 334917 & 0920019 & $10 \mathrm{~S} 09 \mathrm{~W} 23 \mathrm{CDC} 1$ & 63 & 157.08 & 220 & $04-10-97$ & Sparta \\
\hline 335133 & 0921749 & $10 \mathrm{~S} 12 \mathrm{~W} 12 \mathrm{BDD} 1$ & 104 & 116.02 & 220 & $04-09-97$ & Sparta \\
\hline 335623 & 0921251 & 09S11W11CDB1 & 77 & 155.70 & 233 & $04-10-97$ & Sparta \\
\hline 335728 & 0921133 & 09S11W01DCA1 & 39 & 191.50 & 230 & $04-09-97$ & Sparta \\
\hline 335729 & 0921120 & 09S11W01DDA2 & 43 & 206.76 & 250 & $04-09-97$ & Sparta \\
\hline 340133 & 0921539 & $08 \mathrm{~S} 12 \mathrm{~W} 13 \mathrm{CAA} 1$ & 95 & 166.06 & 261 & $04-10-97$ & Sparta \\
\hline \multicolumn{8}{|c|}{ Columbia County } \\
\hline 330136 & 0932622 & $20 \mathrm{~S} 23 \mathrm{~W} 12 \mathrm{AAA} 1$ & 164 & 52.99 & 217 & $03-05-97$ & Sparta \\
\hline 330235 & 0931023 & 19S20W34BDD1 & 77 & 213.16 & 290 & $06-11-97$ & Sparta \\
\hline 330517 & 0931725 & 19S21W 16DBB1 & 108 & 176.19 & 284 & $03-06-97$ & Sparta \\
\hline 330555 & 0931128 & 19S20W09CAC1 & 71 & 260.62 & 332 & $03-06-97$ & Sparta \\
\hline 330557 & 0931146 & 19S20W08DAD1 & 69 & 250.68 & 320 & $03-06-97$ & Sparta \\
\hline 330609 & 0932743 & 19S23W11CDA2 & 195 & 53.38 & 248 & $03-05-97$ & Sparta \\
\hline 331035 & 0931804 & 18S21W17ACD1 & 196 & 119.17 & 315 & $07-09-97$ & Sparta \\
\hline 331039 & 0931255 & 18S20W18ABD1 & -31 & 307.0 & 276 & $06-04-97$ & Sparta \\
\hline 331142 & 0931248 & 18S20W06DDC1 & -12 & 311.58 & 300 & $03-06-97$ & Sparta \\
\hline 331214 & 0931404 & $18 S 21 \mathrm{~W} 01 \mathrm{ACC} 1$ & 0 & 295.23 & 295 & $06-11-97$ & Sparta \\
\hline 331306 & 0930751 & $17 \mathrm{~S} 20 \mathrm{~W} 36 \mathrm{ABC1}$ & 35 & 300.08 & 335 & $06-11-97$ & Sparta \\
\hline 331406 & 0930650 & 17S19W30ABB1 & 30 & 217.97 & 248 & $03-18-97$ & Sparta \\
\hline 331512 & 0930658 & 17S19W18CBD1 & 11 & 293.88 & 305 & $07-08-97$ & Sparta \\
\hline 331516 & 0932303 & 17S22W21ABD1 & 157 & 84.55 & 242 & $05-29-97$ & Sparta \\
\hline 331519 & 0931159 & 17S20W17CDA1 & 16 & 308.69 & 325.10 & $03-07-97$ & Sparta \\
\hline 331519 & 0932136 & 17S22W23BBB1 & 137 & 137.68 & 275 & $05-29-97$ & Sparta \\
\hline 331521 & 0932209 & $17 \mathrm{~S} 22 \mathrm{~W} 22 \mathrm{ABC} 1$ & 114 & 138.10 & 252 & $05-29-97$ & Sparta \\
\hline 331533 & 0930807 & $17 \mathrm{~S} 20 \mathrm{~W} 13 \mathrm{CB} 1$ & -1 & 312.76 & 312 & $03-07-97$ & Sparta \\
\hline 331537 & 0930328 & 17S19W15ABD1 & 39 & 285.86 & 325 & $03-06-97$ & Sparta \\
\hline
\end{tabular}


Table 1. Information pertaining to measured wells completed in the Sparta and Memphis aquifers in Arkansas--Continued

\begin{tabular}{|c|c|c|c|c|c|c|c|}
\hline $\begin{array}{l}\text { Latitude } \\
\text { (degrees) }\end{array}$ & $\begin{array}{c}\text { Longitude } \\
\text { (degrees) }\end{array}$ & Local well number & $\begin{array}{c}\text { Water level } \\
\text { altitude } \\
\text { (feet above } \\
\text { sea level) }\end{array}$ & $\begin{array}{c}\text { Depth to } \\
\text { water } \\
\text { (feet below } \\
\text { land-surface } \\
\text { datum) }\end{array}$ & $\begin{array}{l}\text { Land-surface } \\
\text { datum altitude } \\
\text { (feet above } \\
\text { sea level) }\end{array}$ & $\begin{array}{c}\text { Date of } \\
\text { measure- } \\
\text { ment }\end{array}$ & Aquifer \\
\hline 331607 & 0931818 & 17S21W17BAA1 & 197 & 113.88 & 311 & $05-29-97$ & Sparta \\
\hline 331609 & 0931449 & 17S21W11DCC2 & 2 & 300.52 & 303 & $03-07-97$ & Sparta \\
\hline 331613 & 0931758 & 17S21W08DCA1 & 150 & 148.10 & 298 & $05-29-97$ & Sparta \\
\hline 331742 & 0931423 & 17S21W01 BBC1 & -21 & 326.37 & 305 & $03-18-97$ & Sparta \\
\hline 331753 & 0931513 & $16 \mathrm{~S} 21 \mathrm{~W} 35 \mathrm{CCD} 1$ & 100 & 175.37 & 275 & $07-08-97$ & Sparta \\
\hline 331948 & 0932222 & $16 \mathrm{~S} 22 \mathrm{~W} 22 \mathrm{CCD} 1$ & 209 & 130.78 & 340 & $03-04-97$ & Sparta \\
\hline 332003 & 0930525 & 16S19W20ABD1 & 134 & 160.84 & 295 & $06-12-97$ & Sparta \\
\hline 332041 & 0931622 & $16 \mathrm{~S} 21 \mathrm{~W} 15 \mathrm{CBCl}$ & 76 & 212.32 & 288 & $03-05-97$ & Sparta \\
\hline 332049 & 0931516 & $16 \mathrm{~S} 21 \mathrm{~W} 14 \mathrm{CBB} 1$ & 60 & 221.45 & 281 & $07-09-97$ & Sparta \\
\hline 332117 & 0931139 & 16S20W08DCC1 & 122 & 272.96 & 395 & $07-09-97$ & Spana \\
\hline 332453 & 0931215 & $15 \mathrm{~S} 20 \mathrm{~W} 20 \mathrm{CCB} 1$ & 158 & 213.92 & 372 & $03-05-97$ & Sparta \\
\hline \multicolumn{8}{|c|}{ Craighead County } \\
\hline 354406 & 0904433 & 13N03E23CDD1 & 161 & 86.54 & 248 & $05-15-97$ & Memphis \\
\hline 354751 & 0903130 & $14 \mathrm{~N} 05 \mathrm{E} 36 \mathrm{CBCl}$ & 209 & 10.69 & 220 & $05-15-97$ & Memphis \\
\hline 354839 & 0904033 & 14N04E28DBD1 & 200 & 54.15 & 254 & $05-14-97$ & Memphis \\
\hline 354929 & 0903922 & 14N04E22CBD1 & 202 & 54.28 & 256 & $05-14-97$ & Memphis \\
\hline 355554 & 0902859 & 15N06E18ACA1 & 215 & 15.44 & 230 & $05-15-97$ & Memphis \\
\hline \multicolumn{8}{|c|}{ Crittenden County } \\
\hline 350344 & 0901300 & 05N08E11CCA2 & 191 & 19.97 & 211 & $04-30-97$ & Memphis \\
\hline 350744 & 0900556 & 06N09E23AAB1 & 199 & 22.56 & 222 & $05-01-97$ & Memphis \\
\hline 350958 & 0901738 & 0@N07E01DAD2 & 190 & 18.58 & 209 & $05-01-97$ & Memphis \\
\hline 351349 & 0900628 & 07N09E14BAC1 & 189 & 28.37 & 217 & $05-01-97$ & Memphis \\
\hline \multicolumn{8}{|c|}{ Cross County } \\
\hline 352231 & 0904215 & 09N04E30DCA1 & 172 & 256.97 & 429.32 & $05-02-97$ & Memphis \\
\hline 352359 & 0904514 & 09N03E22AAD1 & 164 & 114.18 & 278 & $05-02-97$ & Memphis \\
\hline 352403 & 0905949 & 09N01E16CAC1 & 157 & 76.68 & 234 & $05-02-97$ & Memphis \\
\hline \multicolumn{8}{|c|}{ Dallas County } \\
\hline 334832 & 0922455 & $10 \mathrm{~S} 13 \mathrm{~W} 34 \mathrm{ACA} 2$ & 124 & 148.07 & 272 & $04-04-97$ & Sparta \\
\hline 335041 & 0923505 & $10 \mathrm{~S} 15 \mathrm{~W} 24 \mathrm{AAB} 1$ & 226 & 16.03 & 242 & $04-04-97$ & Sparta \\
\hline 335304 & 0922413 & 09S13W35CCD1 & 133 & 67.41 & 200 & $04-04-97$ & Sparta \\
\hline 335853 & 0923658 & 08S15W34BDC1 & 212 & 27.67 & 240 & $05-28-97$ & Sparta \\
\hline 335935 & 0924307 & 08S16W27DDD1 & 241 & 31.05 & 272 & $04-04-97$ & Sparta \\
\hline 340429 & 0923332 & 07S14W31AAA1 & 207 & 122.94 & 330 & $04-04-97$ & Sparta \\
\hline 340559 & 0924541 & $07 \mathrm{~S} 16 \mathrm{~W} 20 \mathrm{CAB} 1$ & 296 & 25.56 & 322 & $05-28-97$ & Sparta \\
\hline \multicolumn{8}{|c|}{ Desha County } \\
\hline 333636 & 0912304 & 12S03W34DAD1 & 71 & 75.76 & 147 & $03-24-97$ & Sparta \\
\hline 334605 & 0911705 & $11 \mathrm{~S} 02 \mathrm{~W} 03 \mathrm{CCA} 1$ & 82 & 57.13 & 139 & $03-25-97$ & Sparta \\
\hline 334748 & 0911618 & $10 \mathrm{~S} 02 \mathrm{~W} 26 \mathrm{CCC} 2$ & 85 & 62.84 & 148 & $03-24-97$ & Sparta \\
\hline 335304 & 0913005 & 09S04W28DDD1SP & 56 & 109.09 & 165 & $03-25-97$ & Sparta \\
\hline 335341 & 0911522 & 09S02W26AACl & 89 & 63.59 & 153 & $03-25-97$ & Sparta \\
\hline \multicolumn{8}{|c|}{ Drew County } \\
\hline 332418 & 0912726 & 15S04W 12DDA1 & 68 & 57.03 & 125 & $03-24-97$ & Sparta \\
\hline 333154 & 0913404 & 13S05W36ACB1 & 80 & 89.13 & 169 & $03-21-97$ & Sparta \\
\hline 333649 & 0914400 & 12S06W32DAD1 & 52 & 160.03 & 212 & $03-21-97$ & Sparta \\
\hline 334248 & 0912708 & 11S04W25DAA1 & 68 & 79.67 & 148 & $03-21-97$ & Sparta \\
\hline
\end{tabular}


Table 1. Information pertaining to measured wells completed in the Sparta and Memphis aquifers in Arkansas--Continued

\begin{tabular}{|c|c|c|c|c|c|c|c|}
\hline $\begin{array}{l}\text { Latitude } \\
\text { (degrees) }\end{array}$ & $\begin{array}{l}\text { Longitude } \\
\text { (degrees) }\end{array}$ & Local well number & $\begin{array}{l}\text { Water level } \\
\text { altitude } \\
\text { (feet above } \\
\text { sea level) }\end{array}$ & $\begin{array}{c}\text { Depth to } \\
\text { water } \\
\text { (feet below } \\
\text { land-surface } \\
\text { datum) }\end{array}$ & $\begin{array}{l}\text { Land-surface } \\
\text { datum altitude } \\
\text { (feet above } \\
\text { sea level) }\end{array}$ & $\begin{array}{l}\text { Date of } \\
\text { measure- } \\
\text { ment }\end{array}$ & Aquifer \\
\hline 334601 & 0914121 & 11S06W11DBC1 & 61 & 142.48 & 203 & $03-21-97$ & Sparta \\
\hline 334636 & 0912832 & 11S04W02ACA1 & 65 & 86.52 & 152 & $11-26-97$ & Sparta \\
\hline \multicolumn{8}{|c|}{ Grant County } \\
\hline 340447 & 0921838 & 07S12W27DBC1 & 136 & 98.84 & 235 & $06-11-97$ & Sparta \\
\hline 341024 & 0923545 & 06S15W26ACA1 & 211 & 68.99 & 280 & $04-04-97$ & Sparta \\
\hline 341245 & 0923251 & 06S14W08BDC1 & 179 & 13.93 & 193 & $04-04-97$ & Sparta \\
\hline 341341 & 0921414 & 06S11W05ACA1 & 84 & 196.12 & 280 & $03-31-97$ & Sparta \\
\hline 341812 & 0922653 & 05S13W07ADB1 & 167 & 90.78 & 258 & $06-11-97$ & Sparta \\
\hline 341839 & 0922402 & 05S13W03CDA4 & 174 & 106.64 & 281 & $03-31-97$ & Sparta \\
\hline 341841 & 0923320 & 05S14W06DCC1 & 203 & 90.47 & 293 & $04-04-97$ & Sparta \\
\hline 341845 & 0922359 & 05S13W03DBC1 & 158 & 102.47 & 260 & $03-31-97$ & Sparta \\
\hline 341923 & 0923825 & 05S15W05ABD 1 & 217 & 15.49 & 232 & $04-04-97$ & Sparta \\
\hline 342201 & 0922931 & 04S14W14DCD1 & 166 & 90.62 & 257 & $06-11-97$ & Sparta \\
\hline 342846 & 0922106 & 03S13W12AAA1 & 232 & 129.05 & 361 & $03-31-97$ & Sparta \\
\hline \multicolumn{8}{|c|}{ Hot Spring County } \\
\hline 341456 & 0924150 & 05S16W35ACA1 & 306 & 35.68 & 342 & $04-01-97$ & Sparta \\
\hline \multicolumn{8}{|c|}{ Jefferson County } \\
\hline 340401 & 0915917 & 07S09W35CCB1 & 37 & 232.62 & 270 & $06-03-97$ & Sparta \\
\hline 340547 & 0920420 & 07S10W24CACl & 33 & 278.01 & 311 & $06-03-97$ & Sparta \\
\hline 340631 & 0914520 & 07S07W24BAB1 & 35 & 152.57 & 188 & $06-06-97$ & Sparta \\
\hline 340947 & 0914040 & $06506 \mathrm{~W} 20 \mathrm{CAA} 1$ & 38 & 155.79 & 193.30 & $05-20-97$ & Sparta \\
\hline 341026 & 0915116 & $06 S 08 W 25 A D C 1$ & -6 & 209.80 & 203.48 & $05-29-97$ & Sparta \\
\hline 341054 & 0914125 & 06S06W18DAB1 & 35 & 153.34 & 188 & $05-20-97$ & Sparta \\
\hline 341105 & 0920502 & 06S10W23DBA1 & 14 & 216.17 & 230 & $06-03-97$ & Sparta \\
\hline 341117 & 0920504 & $06510 \mathrm{~W} 23 \mathrm{ACD} 1$ & -2 & 233.83 & 232 & $06-03-97$ & Sparta \\
\hline 341118 & 0920501 & O6S10W11DDB1 & 11 & 217.07 & 228 & $06-03-97$ & Sparta \\
\hline 341138 & 0915516 & $06 \mathrm{~S} 08 \mathrm{~W} 16 \mathrm{CCC} 1$ & -38 & 240.60 & 202.42 & $06-01-97$ & Sparta \\
\hline 341151 & 0920221 & 06509 W $17 \mathrm{CCA} 1$ & -27 & 261.44 & 234.34 & $05-21-97$ & Sparta \\
\hline 341427 & 0915652 & 05S09W35AAB 1 & -71 & 276.00 & 205 & $02-01-97$ & Sparta \\
\hline 341446 & 0915526 & $05 \mathrm{~S} 08 \mathrm{~W} 30 \mathrm{CBA} 1$ & -63 & 270.1 & 207.46 & $05-29-97$ & Sparta \\
\hline 341453 & 0915441 & 05S08W30ADB1 & -55 & 275.82 & 221 & $05-21-97$ & Sparta \\
\hline 341530 & 0915554 & 05S09W24DBD1 & -54 & 261.90 & 208.17 & $05-28-97$ & Sparta \\
\hline 341634 & 0920534 & 05S10W16DBD1 & 32 & 268.20 & 300 & $05-12-97$ & Sparta \\
\hline 341639 & 0920539 & 05S10W16DBB1 & 33 & 282.31 & 315 & $05-21-97$ & Sparta \\
\hline 341658 & 0920546 & 05S10W16BAD1 & 38 & 239.20 & 277 & $05-21-97$ & Sparta \\
\hline 341918 & 0915049 & 04S08W35BBD1 & 2 & 198.45 & 200 & $05-20-97$ & Sparta \\
\hline 342025 & 0920623 & 04S10W29ADB 1 & 64 & 203.98 & 267.55 & $04-11-97$ & Sparta \\
\hline 342107 & 0920440 & 04S10W22BDD 1 & 51 & 193.33 & 244.24 & $04-11-97$ & Sparta \\
\hline 342140 & 0914741 & 04S07W 17BCC1 & 32 & 167.57 & 200 & $05-20-97$ & Sparta \\
\hline 342218 & 0920957 & 04S11W14BADI & 101 & 299.33 & 400 & $04-08-97$ & Sparta \\
\hline 342500 & 0920433 & 03S10W27AAD1 & 110 & 111.55 & 222 & $06-04-97$ & Sparta \\
\hline 342539 & 0920832 & 03S11W25ADC4 & 120 & 192.87 & 313 & $04-08-97$ & Sparta \\
\hline 342618 & 0915455 & 03S08W19BDB1 & 62 & 153.45 & 215 & $05-20-97$ & Sparta \\
\hline 342623 & 0915444 & 03S08W19BAD1 & 56 & 161.36 & 217 & $05-20-97$ & Sparta \\
\hline 342627 & 0915502 & 03S08W19BBD1 & 59 & 156.35 & 215 & $05-20-97$ & Sparta \\
\hline
\end{tabular}


Table 1. Information pertaining to measured wells completed in the Sparta and Memphis aquifers in Arkansas--Continued

\begin{tabular}{|c|c|c|c|c|c|c|c|}
\hline $\begin{array}{l}\text { Latitude } \\
\text { (degrees) }\end{array}$ & $\begin{array}{l}\text { Longitude } \\
\text { (degrees) }\end{array}$ & Local well number & $\begin{array}{l}\text { Water level } \\
\text { altitude } \\
\text { (feet above } \\
\text { sea level) }\end{array}$ & $\begin{array}{c}\text { Depth to } \\
\text { water } \\
\text { (feet below } \\
\text { land-surface } \\
\text { datum) }\end{array}$ & $\begin{array}{l}\text { Land-surface } \\
\text { datum altitude } \\
\text { (feet above } \\
\text { sea level) }\end{array}$ & $\begin{array}{c}\text { Date of } \\
\text { measure- } \\
\text { ment }\end{array}$ & Aquifer \\
\hline 342644 & 0921055 & $03 \mathrm{~S} 11 \mathrm{~W} 22 \mathrm{ABCl}$ & 143 & 166.92 & 310 & $04-11-97$ & Sparta \\
\hline \multicolumn{8}{|c|}{ Lafayette County } \\
\hline 330223 & 0933033 & 20S23W05ADB 1 & 204 & 38.42 & 242 & $03-04-97$ & Sparta \\
\hline 330351 & 0933103 & 19S23W29BDB 1 & 210 & 40.24 & 250 & $03-04-97$ & Sparta \\
\hline 330546 & 0933916 & $19 \mathrm{~S} 25 \mathrm{~W} 13 \mathrm{CAB} 1$ & 228 & 27.03 & 255 & $03-04-97$ & Sparta \\
\hline 330911 & 0933038 & $18 \mathrm{~S} 23 \mathrm{~W} 29 \mathrm{ACC} 1$ & 244 & 10.72 & 255 & $03-04-97$ & Sparta \\
\hline 331523 & 0932952 & 17S23W21BCB1 & 257 & 38.36 & 295 & $03-04-97$ & Sparta \\
\hline 331526 & 0933402 & 17S24W23BBD1 & 239 & 21.61 & 261 & $03-04-97$ & Sparta \\
\hline 332026 & 0933728 & 16S24W19DBC1 & 220 & 45.32 & 265 & $03-03-97$ & Sparta \\
\hline 332140 & 0932611 & 16S23W12CAD1 & 255 & 67.29 & 322 & $03-05-97$ & Sparta \\
\hline \multicolumn{8}{|c|}{ Lee County } \\
\hline 345011 & 0904749 & 03N03E28CDB1 & 159 & 47.57 & 207 & $04-30-97$ & Sparta \\
\hline \multicolumn{8}{|c|}{ Lincoln County } \\
\hline 335229 & 0913758 & 10S05W05ADB1 & 62 & 108.77 & 171 & $04-09-97$ & Sparta \\
\hline 335631 & 0915121 & 09S07W07DAD1 & 34 & 265.91 & 300 & $04-09-97$ & Sparta \\
\hline 335907 & 0913333 & 08S05W35ACC1 & 52 & 112.64 & 165 & $04-11-97$ & Sparta \\
\hline 335955 & 0915301 & 08S08W35DBB1 & 34 & 205.58 & 240 & $04-09-97$ & Sparta \\
\hline 335957 & 0914352 & 08S06W31DCC1 & 27 & 122.98 & 150 & $05-21-97$ & Sparta \\
\hline 340200 & 0912800 & 08S04W22AAA1 & 59 & 80.57 & 140 & $04-11-97$ & Sparta \\
\hline 340345 & 0913446 & 08S05W03BAA2 & 50 & 129.73 & 180 & $04-11-97$ & Sparta \\
\hline 340444 & 0915042 & 07S07W30CDC1 & 37 & 170.58 & 208 & $04-10-97$ & Sparta \\
\hline \multicolumn{8}{|c|}{ Lonoke County } \\
\hline 343227 & 0915227 & 02S08W16BDA1 & 99 & 117.06 & 216 & $03-27-97$ & Sparta \\
\hline 344425 & 0914503 & 01N07W03BCCl & 106 & 117.19 & 223 & $04-17-97$ & Sparta \\
\hline 344448 & 0914618 & 02N07W32DDD1 & 108 & 118.37 & 226 & $06-12-97$ & Sparta \\
\hline 344609 & 0914322 & 02N07W23BAA1 & 101 & 134.51 & 236 & $04-24-97$ & Sparta \\
\hline 344652 & 0914419 & 02NO7W22DBAI & 110 & 117.47 & 227 & $04-17-97$ & Sparta \\
\hline 344702 & 0914149 & 02N07W24DAC1 & 103 & 128.22 & 231 & $04-14-97$ & Sparta \\
\hline 344906 & 0914500 & 02N07W09AAA1 & 138 & 94.04 & 232 & $06-09-97$ & Sparta \\
\hline 344940 & 0914721 & 02N07W06ACD1 & 124 & 116.88 & 241 & $06-09-97$ & Sparta \\
\hline 345152 & 0915025 & 03N08W23DDD1 & 138 & 96.00 & 234 & $04-14-97$ & Sparta \\
\hline 345444 & 0914426 & 03N07W03CAA1 & 160 & 75.34 & 235 & $06-09-97$ & Memphis \\
\hline \multicolumn{8}{|c|}{ Miller County } \\
\hline 331604 & 0934406 & 17S25W18CDB1 & 210 & 10.03 & 220 & 03-03-97 & Sparta \\
\hline \multicolumn{8}{|c|}{ Monroe County } \\
\hline 344145 & 0911756 & $01 N 03 W 14 C C B 1$ & 108 & 64.00 & 172 & $04-28-97$ & Sparta \\
\hline 345043 & 0911026 & 03N02W26DAB1 & 150 & 42.05 & 192 & $05-19-97$ & Sparta \\
\hline 345535 & 0911221 & 04N02W28DDD4 & 166 & 26.47 & 192 & $04-18-97$ & Sparta \\
\hline 345618 & 0911509 & 04N02W30BACl & 162 & 18.11 & 180 & $04-18-97$ & Sparta \\
\hline \multicolumn{8}{|c|}{ Nevada County } \\
\hline 332818 & 0931740 & 14S21W32DCD1 & 257 & 113.23 & 370 & $03-05-97$ & Sparta \\
\hline 333324 & 0930708 & 13S20W36DCC1 & 244 & 105.84 & 350 & $03-05-97$ & Sparta \\
\hline \multicolumn{8}{|c|}{ Ouachita County } \\
\hline 332305 & 0925434 & 15S18W36ADD1 & 66 & 94.09 & 160 & $06-10-97$ & Sparta \\
\hline 332437 & 0930431 & $15 S 19 W 21 C D D 2$ & 136 & 144.04 & 280 & $07-08-97$ & Sparta \\
\hline
\end{tabular}


Table 1. Information pertaining to measured wells completed in the Sparta and Memphis aquifers in Arkansas--Continued

\begin{tabular}{|c|c|c|c|c|c|c|c|}
\hline $\begin{array}{l}\text { Latitude } \\
\text { (degrees) }\end{array}$ & $\begin{array}{c}\text { Longitude } \\
\text { (degrees) }\end{array}$ & Local well number & $\begin{array}{l}\text { Water level } \\
\text { altitude } \\
\text { (feet above } \\
\text { sea level) }\end{array}$ & $\begin{array}{c}\text { Depth to } \\
\text { water } \\
\text { (feet below } \\
\text { land-surface } \\
\text { datum) }\end{array}$ & $\begin{array}{l}\text { Land-surface } \\
\text { datum altitude } \\
\text { (feet above } \\
\text { sea level) }\end{array}$ & $\begin{array}{c}\text { Date of } \\
\text { measure- } \\
\text { ment }\end{array}$ & Aquifer \\
\hline 332618 & 0930318 & 15S19W10DCC1 & 144 & 65.58 & 210 & $03-18-97$ & Sparta \\
\hline 332804 & 0925251 & 14S17W32CAD1 & 135 & 84.97 & 220 & $06-10-97$ & Sparta \\
\hline 332942 & 0930513 & $14 S 19 W 29 A B B 1$ & 194 & 86.18 & 280 & $03-11-97$ & Sparta \\
\hline 333234 & 0925252 & 14S1TW05CAD 1 & 121 & 35.83 & 157 & $03-10-97$ & Sparta \\
\hline 333252 & 0924926 & 14S1TW02ABB 1 & 9 & 110.94 & 120 & $03-17-97$ & Sparta \\
\hline 333435 & 0930417 & $13 S 19 \mathrm{~W} 28 \mathrm{BCD} 1$ & 193 & 37.24 & 230 & $03-11-97$ & Sparta \\
\hline 333934 & 0924206 & $12 S 16 \mathrm{~W} 26 \mathrm{ABD} 1$ & 94 & 39.63 & 134 & $03-10-97$ & Sparta \\
\hline 333942 & 0924252 & 12S16W25BDA1 & 102 & 34.78 & 137 & $03-10-97$ & Sparta \\
\hline 334018 & 0925948 & $12 S 18 W 19 \mathrm{CDC} 1$ & 204 & 30.56 & 235 & $03-11-97$ & Sparta \\
\hline 334154 & 0930109 & 12S19W14AAA1 & 226 & 10.54 & 237 & $06-10-97$ & Sparta \\
\hline 334218 & 0923914 & $12 \mathrm{~S} 15 \mathrm{~W} 09 \mathrm{BBA} 1$ & 159 & 54.20 & 213 & $05-30-97$ & Sparta \\
\hline 334251 & 0930351 & 12S19W09BAB 1 & 276 & 13.95 & 290 & $03-11-97$ & Sparta \\
\hline 334342 & 0924835 & $11 S 17 W 36 C C A 1$ & 129 & 3.66 & 133 & $03-10-97$ & Sparta \\
\hline 334443 & 0923725 & $11 \mathrm{~S} 15 \mathrm{~W} 27 \mathrm{ABD} 1$ & 134 & 66.22 & 200 & $03-10-97$ & Sparta \\
\hline 334614 & 0925759 & 11S18W20AAA1 & 270 & 30.86 & 301 & $03-11-97$ & Sparta \\
\hline \multicolumn{8}{|c|}{ Phillips County } \\
\hline 341822 & 0905124 & 04S02E $25 \mathrm{CCCl}$ & 131 & 35.24 & 166 & $04-29-97$ & Sparta \\
\hline 342403 & 0904914 & 03S03E30DAA1 & 137 & 34.65 & 172 & $04-29-97$ & Sparta \\
\hline 342754 & 0903621 & 03S05E05BAB 1 & 143 & 36.99 & 180 & $04-30-97$ & Sparta \\
\hline 342856 & 0903636 & $02 \mathrm{~S} 05 \mathrm{E} 29 \mathrm{CCC} 1$ & 157 & 21.52 & 179 & $04-29-97$ & Sparta \\
\hline 343110 & 0903525 & 02S05E $16 \mathrm{BCB} 1$ & 162 & 28.25 & 190 & $04-29-97$ & Sparta \\
\hline 343242 & 0903902 & 02S04E02DBA1 & 126 & 123.54 & 250 & $04-29-97$ & Sparta \\
\hline 343322 & 0905056 & $02 \mathrm{~S} 02 \mathrm{E} 01 \mathrm{ADCl}$ & 141 & 34.92 & 176 & $04-29-97$ & Sparta \\
\hline 343324 & 0905446 & $01 \mathrm{~S} 02 \mathrm{E} 32 \mathrm{DDC} 1$ & 134 & 77.46 & 211 & $04-28-97$ & Sparta \\
\hline \multicolumn{8}{|c|}{ Poinsett County } \\
\hline 352724 & 0905846 & $10 \mathrm{~N} 01 \mathrm{E} 27 \mathrm{CCl}$ & 153 & 77.86 & 231 & $05-13-97$ & Memphis \\
\hline 352844 & 0904433 & 10N03E23CACl & 154 & 104.08 & 258 & $05-13-97$ & Memphis \\
\hline 352930 & 0905825 & 10N01E15DBB1 & 147 & 84.99 & 232 & $05-13-97$ & Memphis \\
\hline 353144 & 0904454 & 10N03E02BCD1 & 149 & 102.09 & 251 & $05-14-97$ & Memphis \\
\hline 353225 & 0904316 & 11 N03E25ACC1 & 150 & 123.03 & 273 & $05-13-97$ & Memphis \\
\hline 353448 & 0905330 & $11 \mathrm{~N} 02 \mathrm{E} 16 \mathrm{CCCl}$ & 149 & 94.29 & 243 & $05-13-97$ & Memphis \\
\hline 353736 & 0904354 & 12N03E35DDA1 & 146 & 101.34 & 247 & $05-13-97$ & Memphis \\
\hline 354124 & 0904917 & $12 \mathrm{NO2E} 12 \mathrm{DDCl}$ & 153 & 94.67 & 248 & $05-13-97$ & Memphis \\
\hline \multicolumn{8}{|c|}{ Prairie County } \\
\hline 343639 & 0913352 & $01 S 05 W 20 A B B 1$ & 74 & 146.34 & 220 & $03-27-97$ & Sparta \\
\hline 343748 & 0913654 & 01S06W11DBD1 & 77 & 148.90 & 226 & $03-27-97$ & Sparta \\
\hline 343902 & 0913531 & $01505 W 06 \mathrm{BCB} 1$ & 76 & 144.04 & 220 & $03-27-97$ & Sparta \\
\hline 344113 & 0913504 & $01 \mathrm{~N} 05 \mathrm{~W} 19 \mathrm{CDCl}$ & 84 & 128.16 & 212 & $03-27-97$ & Sparta \\
\hline 344136 & 0914145 & 01N06W19BCD1 & 101 & 121.63 & 223 & $06-10-97$ & Sparta \\
\hline 344444 & 0913658 & 01N06W02ABB1 & 117 & 106.14 & 223 & $04-17-97$ & Sparta \\
\hline 344644 & 0913828 & 02N06W21DAD1 & 115 & 117.19 & 232 & $04-17-97$ & Sparta \\
\hline 344649 & 0912801 & 02N04W19ACB1 & 126 & 85.33 & 211 & $04-17-97$ & Sparta \\
\hline 344653 & 0913800 & 02N06W22BDD1 & 112 & 120.77 & 233 & $04-17-97$ & Sparta \\
\hline 344704 & 0914033 & 02N06W20BCB1 & 106 & 129.65 & 236 & $04-17-97$ & Sparta \\
\hline 344906 & 0913837 & 02N06W04DBD1 & 136 & 96.66 & 233 & $06-12-97$ & Memphis \\
\hline
\end{tabular}


Table 1. Information pertaining to measured wells completed in the Sparta and Memphis aquifers in Arkansas--Continued

\begin{tabular}{|c|c|c|c|c|c|c|c|}
\hline $\begin{array}{l}\text { Latitude } \\
\text { (degrees) }\end{array}$ & $\begin{array}{l}\text { Longitude } \\
\text { (degrees) }\end{array}$ & Local well number & $\begin{array}{l}\text { Water level } \\
\text { altitude } \\
\text { (feet above } \\
\text { sea level) }\end{array}$ & $\begin{array}{c}\text { Depth to } \\
\text { water } \\
\text { (feet below } \\
\text { land-surface } \\
\text { datum) }\end{array}$ & $\begin{array}{l}\text { Land-surface } \\
\text { datum altitude } \\
\text { (feet above } \\
\text { sea level) }\end{array}$ & $\begin{array}{c}\text { Date of } \\
\text { measure- } \\
\text { ment }\end{array}$ & Aquifer \\
\hline 345140 & 0914004 & 03N06W20CDD1 & 130 & 95.22 & 225 & $06-12-97$ & Memphis \\
\hline 345144 & 0913356 & 03N05W20CCC1 & 113 & 99.92 & 213 & $06-12-97$ & Memphis \\
\hline 345451 & 0913042 & 03N05W03ADA1 & 107 & 97.86 & 205 & $06-12-97$ & Memphis \\
\hline \multicolumn{8}{|c|}{ Pulaski County } \\
\hline 344203 & 0920818 & $01 N 11 W 24 A C D 1$ & 210 & 27.10 & 237 & $04-15-97$ & Sparta \\
\hline \multicolumn{8}{|c|}{ St. Francis County } \\
\hline 345705 & 0902842 & 04N06E16CCB1 & 154 & 43.77 & 198 & $04-25-97$ & Memphis \\
\hline 345746 & 0904320 & 04N04E18BAB1 & 156 & 63.57 & 220 & $04-25-97$ & Memphis \\
\hline \multicolumn{8}{|c|}{ Saline County } \\
\hline 342904 & 0923222 & 03S14W05CCC1 & 307 & 7.91 & 315 & $04-14-97$ & Sparta \\
\hline \multicolumn{8}{|c|}{ Union County } \\
\hline 330110 & 0924321 & 19S16W35DDC1 & -48 & 222.96 & 175 & $03-12-97$ & Sparta \\
\hline 330219 & 0921112 & 19S11W25AAA1 & -1 & 136.00 & 135 & 03-12-97 & Sparta \\
\hline 330327 & 0920905 & 19S10W 16CBC1 & 7 & 74.86 & 82 & $03-12-97$ & Sparta \\
\hline 330631 & 0923708 & 18S15W35DAC1 & -104 & 305.39 & 201 & $03-12-97$ & Sparta \\
\hline 330652 & 0922119 & 18S12W33BBB1 & -13 & 125.47 & 112 & $03-12-97$ & Sparta \\
\hline 330657 & 0923859 & 18S15W33ADA1 & -115 & 368.00 & 253 & 03-12-97 & Sparta \\
\hline 330807 & 0924613 & 18S16W28BBB1 & -115 & 339.88 & 225 & $03-07-97$ & Spanta \\
\hline 330855 & 0925056 & 18S17W22BDD1 & -78 & 362.67 & 285 & $03-06-97$ & Sparta \\
\hline 331006 & 0925448 & 18S17W18BBD1 & -30 & 300.27 & 270 & $03-06-97$ & Sparta \\
\hline 331011 & 0924317 & 18S16W11DAB1 & -147 & 416.72 & 270 & $03-07-97$ & Sparta \\
\hline 331024 & 0924229 & $18 \mathrm{~S} 16 \mathrm{~W} 12 \mathrm{ACB} 1$ & -167 & 469.86 & 303 & 03-07-97 & Sparta \\
\hline 331040 & 0923531 & 18S14W06CCA1 & -146 & 371.00 & 225 & $03-12-97$ & Sparta \\
\hline 331057 & 0925559 & 18S18W11ACA1 & 0 & 244.64 & 245 & $03-06-97$ & Sparta \\
\hline 331142 & 0924118 & 17S15W31DCA1 & -145 & 416.74 & 272 & $03-12-97$ & Sparta \\
\hline 331203 & 0922218 & 17S12W32BBC1 & -7 & 236.98 & 230 & $03-12-97$ & Sparta \\
\hline 331205 & 0922916 & 17S13W31BAC1 & -68 & 284.13 & 216 & 03-13-97 & Sparta \\
\hline 331228 & 0924038 & $17 \mathrm{~S} 15 \mathrm{~W} 29 \mathrm{CDC} 1$ & -198 & 418.44 & 220 & $03-19-97$ & Sparta \\
\hline 331300 & 0925356 & 17S17W30DCD1 & -29 & 309.28 & 280 & $03-19-97$ & Sparta \\
\hline 331358 & 0924248 & 17S16W24BDB1 & -199 & 403.57 & 205 & $03-19-97$ & Sparta \\
\hline 331438 & 0924119 & 17S15W18DBB1 & -177 & 359.74 & 182.93 & $05-19-97$ & Sparta \\
\hline 331805 & 0925709 & $16 S 18 W 34 A B C 2$ & 59 & 189.50 & 248 & 03-19-97 & Sparta \\
\hline 331900 & 0923956 & 16S15W20DAA1 & -75 & 264.87 & 190 & $03-18-97$ & Sparta \\
\hline 331944 & 0923217 & $16 \mathrm{~S} 14 \mathrm{~W} 15 \mathrm{CAB} 1$ & -56 & 150.04 & 94 & 03-19-97 & Sparta \\
\hline 332205 & 0924330 & 16S16W02ABC1 & -55 & 170.83 & 116 & $03-18-97$ & Sparta \\
\hline \multicolumn{8}{|c|}{ Woodruff County } \\
\hline 350026 & 0911454 & 05N02W31DCB3 & 178 & 15.13 & 193 & $04-18-97$ & Memphis \\
\hline 350310 & 0910727 & 05N01W17DBB1 & 169 & 40.81 & 210 & $04-18-97$ & Memphis \\
\hline 350426 & 0910406 & 05N01W11ABA1 & 160 & 51.46 & 211 & $04-18-97$ & Memphis \\
\hline 351932 & 0910310 & 08N01W12CDA1 & 155 & 70.18 & 225 & $05-16-97$ & Memphis \\
\hline
\end{tabular}


Table 2. Information pertaining to measured wells completed in the Sparta aquifer in Louisiana

\begin{tabular}{|c|c|c|c|c|c|c|c|}
\hline $\begin{array}{l}\text { Latitude } \\
\text { (degrees) }\end{array}$ & $\begin{array}{l}\text { Longitude } \\
\text { (degrees) }\end{array}$ & $\begin{array}{l}\text { Local well } \\
\text { number }\end{array}$ & $\begin{array}{l}\text { Water level } \\
\text { altitude } \\
\text { (feet above } \\
\text { sea level) }\end{array}$ & $\begin{array}{l}\text { Depth to } \\
\text { water } \\
\text { (feet below } \\
\text { land-surface } \\
\text { datum) }\end{array}$ & $\begin{array}{c}\text { Land-surface } \\
\text { datum altitude } \\
\text { (feet above } \\
\text { sea level) }\end{array}$ & $\begin{array}{l}\text { Date of } \\
\text { measure- } \\
\text { ment }\end{array}$ & Aquifer \\
\hline \multicolumn{8}{|c|}{ Bienville Parish } \\
\hline 321733 & 0930350 & $\mathrm{Bi}-76$ & 230 & 50.46 & 280 & $10-01-96$ & Sparta \\
\hline 321402 & 0925457 & $\mathrm{Bi}-100$ & 187 & 33.21 & 220 & $10-16-96$ & Sparta \\
\hline 321709 & 0925239 & $\mathrm{Bi}-112$ & 99 & 115.82 & 215 & $10-01-96$ & Sparta \\
\hline 323505 & 0925350 & $\mathrm{Bi}-144$ & 84 & 236.42 & 320 & $10-01-96$ & Sparta \\
\hline 322436 & 0925005 & $\mathrm{Bi}-166$ & 79 & 181.43 & 260 & $10-01-96$ & Sparta \\
\hline 321101 & 0925221 & $\mathrm{Bi}-186$ & 138 & 41.97 & 180 & $10-16-96$ & Sparta \\
\hline 321538 & 0930016 & $\mathrm{Bi}-192$ & 211 & 74.49 & 285 & $10-16-96$ & Sparta \\
\hline 322119 & 0925723 & $\mathrm{Bi}-216$ & 183 & 16.70 & 200 & $10-01-96$ & Sparta \\
\hline \multicolumn{8}{|c|}{ Caldwell Parish } \\
\hline 320154 & 0921646 & $\mathrm{Ca}-86 \mathrm{~B}$ & 81 & 79.28 & 160 & $10-01-96$ & Sparta \\
\hline \multicolumn{8}{|c|}{ Claiborne Parish } \\
\hline 325752 & 0930827 & $\mathrm{Cl}-9$ & 90 & 270.01 & 360 & $10-09-96$ & Sparta \\
\hline 324707 & 0930250 & $\mathrm{Cl}-58$ & 110 & 140.01 & 250 & $10-09-96$ & Sparta \\
\hline 324817 & 0925125 & $\mathrm{Cl}-111$ & 45 & 255.46 & 300 & $10-08-96$ & Sparta \\
\hline 325228 & 0924902 & $\mathrm{Cl}-116$ & -15 & 259.73 & 245 & $10-10-96$ & Sparta \\
\hline 325437 & 0925033 & $\mathrm{Cl}-148$ & -7 & 196.80 & 190 & $10-10-96$ & Sparta \\
\hline 330002 & 0924459 & $\mathrm{Cl}-149$ & -64 & 294.36 & 230 & $10-10-96$ & Sparta \\
\hline \multicolumn{8}{|c|}{ Jackson Parish } \\
\hline 321709 & 0924524 & $\mathrm{Ja}-49$ & -41 & 201.19 & 160 & $10-02-96$ & Sparta \\
\hline 322357 & 0923417 & $\mathrm{Ja}-147$ & -26 & 246.47 & 220 & $10-16-96$ & Sparta \\
\hline 321338 & 0923458 & $\mathrm{Ja}-148$ & 20 & 225.15 & 245 & $10-02-96$ & Sparta \\
\hline \multicolumn{8}{|c|}{ Lincoln Parish } \\
\hline 324141 & 0923905 & L-26 & -25 & 180.27 & 155 & $10-10-96$ & Sparta \\
\hline 323458 & 0922751 & L-68 & -70 & 249.60 & 180 & $10-10-96$ & Sparta \\
\hline 323013 & 0924820 & $\mathrm{~L}-113$ & 37 & 317.56 & 355 & $10-02-96$ & Sparta \\
\hline 324202 & 0923226 & $\mathrm{~L}-117$ & -52 & 137.85 & 86 & $10-15-96$ & Sparta \\
\hline \multicolumn{8}{|c|}{ Morehouse Parish } \\
\hline 324626 & 0915439 & Mo-5 & -36 & 153.52 & 117 & $10-04-96$ & Sparta \\
\hline 324753 & 0914712 & Mo-342 & -2 & 89.85 & 88 & $10-03-96$ & Sparta \\
\hline 325707 & 0915747 & Mo-350 & 7 & 105.56 & 112 & $10-03-96$ & Sparta \\
\hline \multicolumn{8}{|c|}{ Ouachita Parish } \\
\hline 322843 & 0920844 & $\mathrm{Ou}-80$ & -224 & 283.76 & 60 & $10-10-96$ & Sparta \\
\hline 322422 & 0920207 & $\mathrm{Ou}-401 \mathrm{~A}$ & -44 & 106.13 & 62 & $10-10-96$ & Sparta \\
\hline 321714 & 0920414 & $\mathrm{Ou}-402$ & 4 & 58.98 & 63 & $10-10-96$ & Sparta \\
\hline 323030 & 0915548 & $\mathrm{Ou}-404$ & -19 & 80.32 & 61 & $10-15-96$ & Sparta \\
\hline 322531 & 0920539 & Ou-406 & -91 & 157.25 & 67 & $10-10-96$ & Sparta \\
\hline 323100 & 0921658 & Ou-444 & -111 & 229.09 & 118 & $10-23-96$ & Sparta \\
\hline 322437 & 0922431 & $\mathrm{Ou}-488$ & -53 & 332.73 & 280 & $10-23-96$ & Sparta \\
\hline
\end{tabular}


Table 2. Information pertaining to measured wells completed in the Sparta aquifer in Louisiana--Continued

\begin{tabular}{|c|c|c|c|c|c|c|c|}
\hline $\begin{array}{l}\text { Latitude } \\
\text { (degrees) }\end{array}$ & $\begin{array}{c}\text { Longitude } \\
\text { (degrees) }\end{array}$ & $\begin{array}{l}\text { Local well } \\
\text { number }\end{array}$ & $\begin{array}{l}\text { Water level } \\
\text { altitude } \\
\text { (feet above } \\
\text { sea level) }\end{array}$ & $\begin{array}{c}\text { Depth to } \\
\text { water } \\
\text { (feet below } \\
\text { land-surface } \\
\text { datum) }\end{array}$ & $\begin{array}{c}\text { Land-surface } \\
\text { datum altitude } \\
\text { (feet above } \\
\text { sea level) }\end{array}$ & $\begin{array}{c}\text { Date of } \\
\text { measure- } \\
\text { ment }\end{array}$ & Aquifer \\
\hline \multicolumn{8}{|c|}{ Union Parish } \\
\hline 324415 & 0920902 & Un-26 & -50 & 184.00 & 134 & $10-04-96$ & Sparta \\
\hline 325028 & 0921133 & Un-78 & -34 & 208.60 & 175 & $10-24-96$ & Sparta \\
\hline 324955 & 0920840 & Un-79 & -35 & 153.40 & 118 & $10-24-96$ & Sparta \\
\hline 325550 & 0923916 & Un-83 & -52 & 174.00 & 122 & $10-16-96$ & Sparta \\
\hline 325647 & 0922415 & Un-84 & -39 & 248.70 & 210 & $10-24-96$ & Sparta \\
\hline 325929 & 0921140 & Un-86 & -11 & 100.63 & 90 & $10-16-96$ & Sparta \\
\hline 323655 & 0922117 & Un-134 & -87 & 307.13 & 220 & $10-23-96$ & Sparta \\
\hline \multicolumn{8}{|c|}{ Webster Parish } \\
\hline 325948 & 0932720 & Wb-127 & 209 & 61.37 & 270 & $10-21-96$ & Sparta \\
\hline 324919 & 0932507 & Wb-164 & 156 & 64.32 & 220 & $10-07-96$ & Sparta \\
\hline 323220 & 0931659 & Wb-219 & 185 & 5.17 & 190 & $10-08-96$ & Sparta \\
\hline 323221 & 0931404 & $\mathrm{~Wb}-271$ & 194 & 84.25 & 278 & $10-09-96$ & Sparta \\
\hline 323553 & 0931411 & Wb-285 & 159 & 180.68 & 340 & $10-08-96$ & Sparta \\
\hline 325200 & 0931517 & Wb-326 & 145 & 114.51 & 260 & $10-04-96$ & Sparta \\
\hline 330040 & 0931900 & Wb-338 & 135 & 94.89 & 230 & $10-04-96$ & Sparta \\
\hline 323630 & 0931736 & Wb-349 & 110 & 70.38 & 180 & $10-07-96$ & Sparta \\
\hline 323423 & 0931459 & Wb-359 & 200 & 79.68 & 280 & $10-09-96$ & Sparta \\
\hline 325518 & 0932219 & Wb-399 & 160 & 45.24 & 205 & $10-04-96$ & Sparta \\
\hline 323534 & 0932005 & Wb-415 & 143 & 17.20 & 160 & $10-09-96$ & Sparta \\
\hline \multicolumn{8}{|c|}{ Winn Parish } \\
\hline 315527 & 0923708 & W-28 & 53 & 52.14 & 105 & $10-01-96$ & Sparta \\
\hline 315450 & 0923101 & W-144B & 102 & 37.55 & 140 & $10-01-96$ & Sparta \\
\hline 320541 & 0922916 & W-172 & 49 & 91.21 & 140 & $10-01-96$ & Sparta \\
\hline 315338 & 0924000 & W-177 & 132 & 53.34 & 185 & $10-01-96$ & Sparta \\
\hline 315948 & 0923003 & W-179 & 86 & 108.70 & 195 & $10-01-96$ & Sparta \\
\hline
\end{tabular}

adjoining counties, Arkansas and Prairie Counties. The cones of depression in Columbia and Union Counties are elongated to the southeast and northwest because of heavy industrial pumpage, respectively, and coalesce at or near the Columbia and Union County line. In Louisiana, the potentiometric surface of the Sparta aquifer contains a cone of depression descending below sea level in the north-central parts of the State. The cone of depression in Ouachita Parish is elongated to the northwest and coalesces with the Union County cone of depression near the Arkansas-Louisiana State line. There are several smaller cones of depression throughout the study area, which represent localized pumpage of one or two wells that do not influence water levels on a regional scale.

\section{LONG-TERM HYDROGRAPHS}

Twenty-five years of water-level data from each of 10 selected wells completed in the Sparta and Memphis aquifers were plotted to illustrate the history of water-levels in selected areas of Arkansas and Louisiana (fig. 2). During the period 1972-1997, water-level declines were less than 0.8 feet per year $(\mathrm{ft} / \mathrm{yr})$ in Columbia and Phillips Counties, between 1.0 and 1.5 $\mathrm{ft} / \mathrm{yr}$ in Arkansas, Bradley, Desha, Jefferson, Poinsett, and Union Counties, and more than $2.0 \mathrm{ft} / \mathrm{yr}$ in Lincoln and Ouachita Parishes. Two hydrographs from Louisiana with a shorter period of record (1979-1997) illustrate that the water level in a Claiborne Parish well has decreased more than $1.5 \mathrm{ft} / \mathrm{yr}$ and that the water level in a Webster Parish well has fluctuated slightly since 1979. 


\section{A. ARKANSAS COUNTY 03S04W02CCB1}

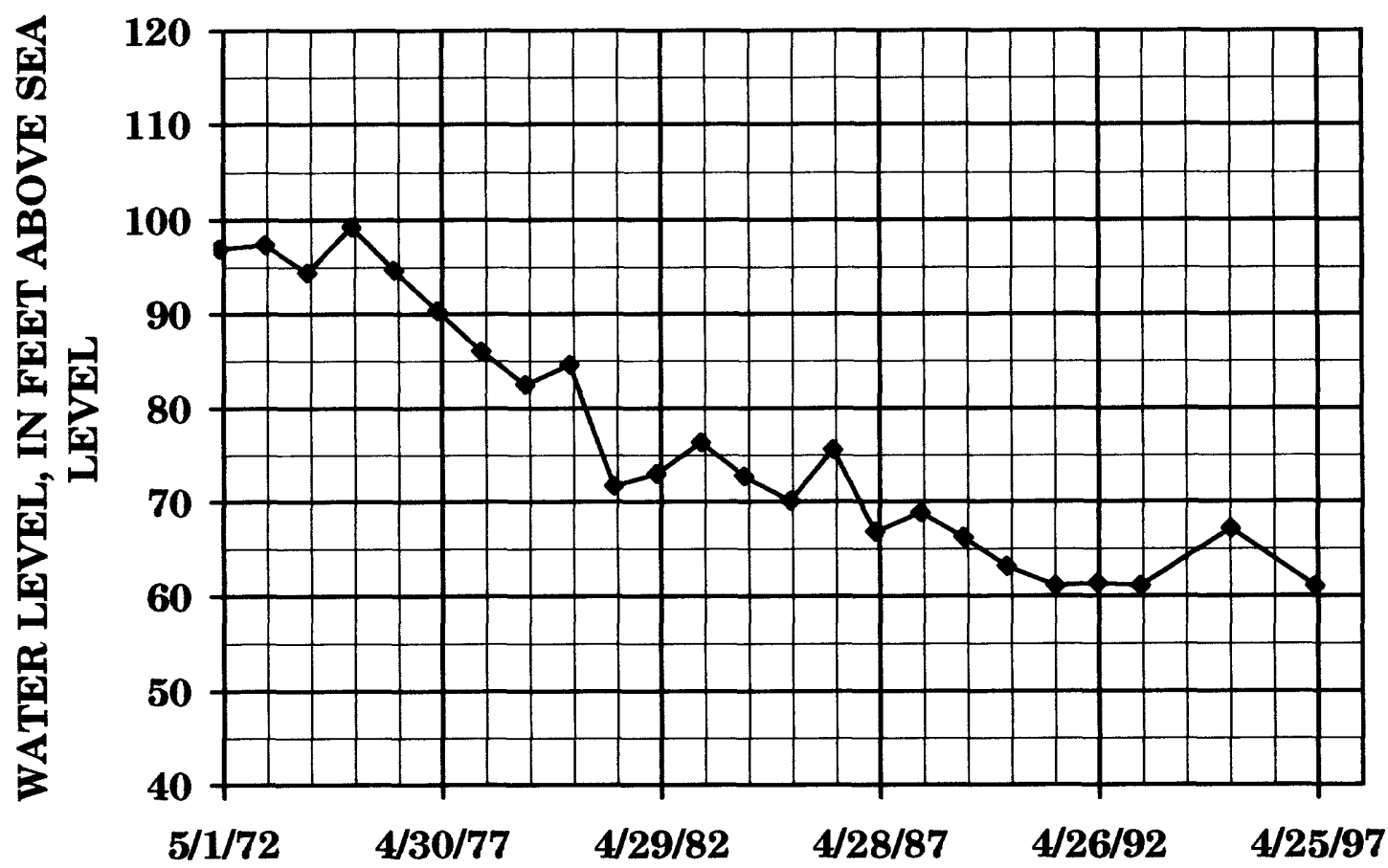

B. BRADLEY COUNTY 16S12W21CAA1

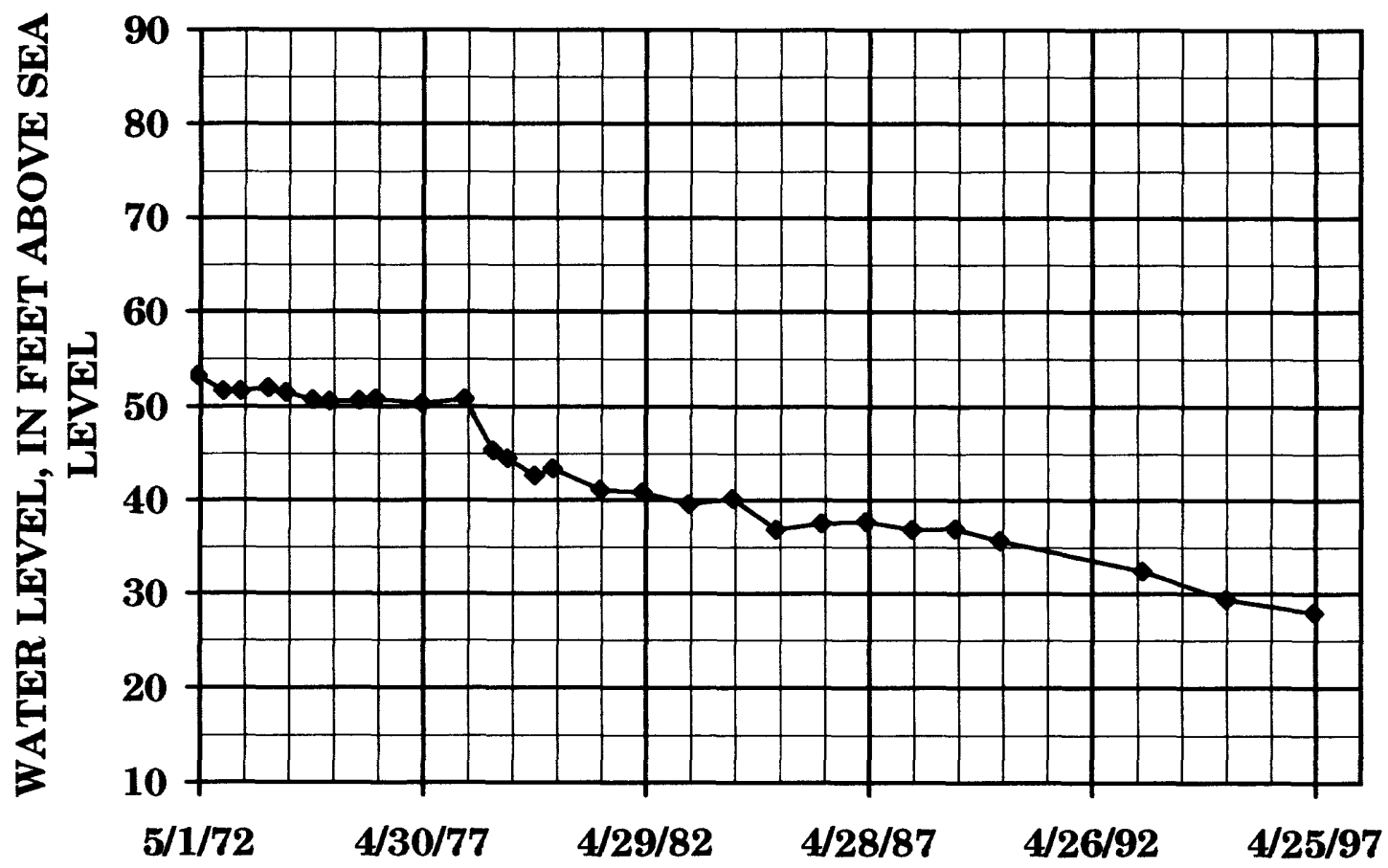

Figure 2. Water-level hydrographs for selected wells completed in the Sparta and Memphis aquifers (page 1 of 6). 


\section{COLUMBIA COUNTY 18S20W06DDC1}
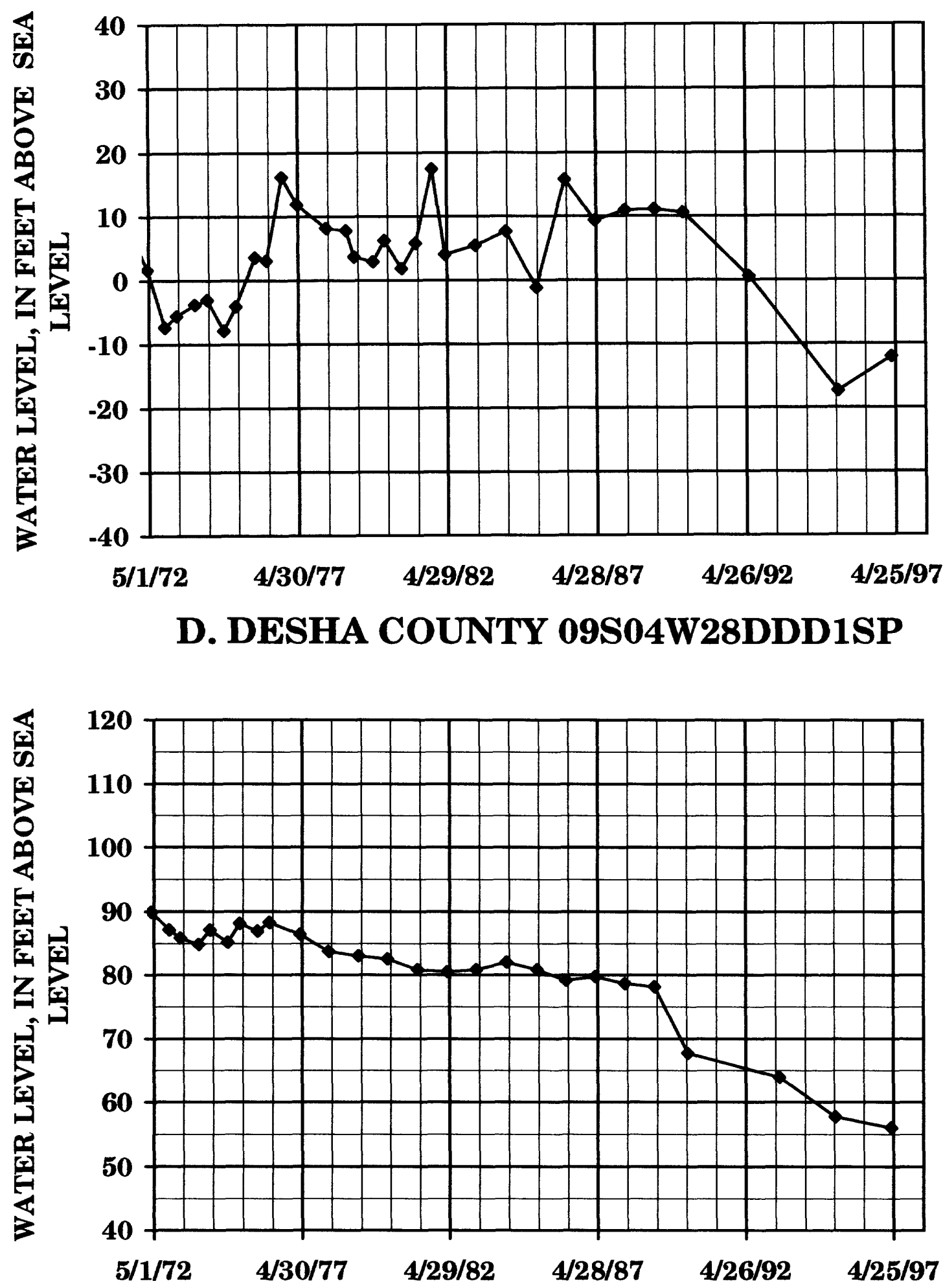

Flgure 2. Water-level hydrographs for selected wells completed in the Sparta and Memphis aquifers (page 2 of 6). 


\section{E. JEFFERSON COUNTY 06S08W16CCC1}

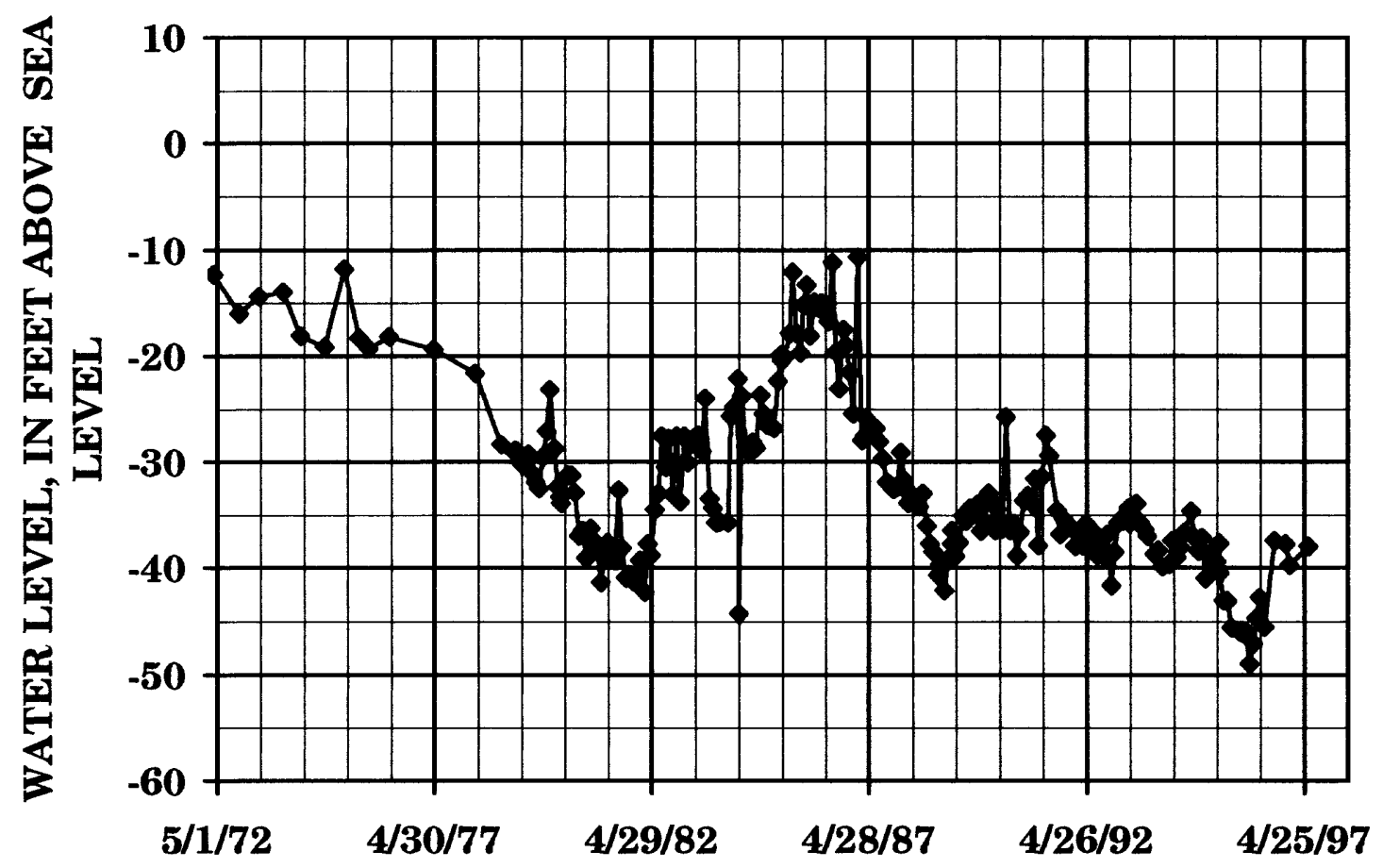

F. PHILLIPS COUNTY 01S02E32DDC1

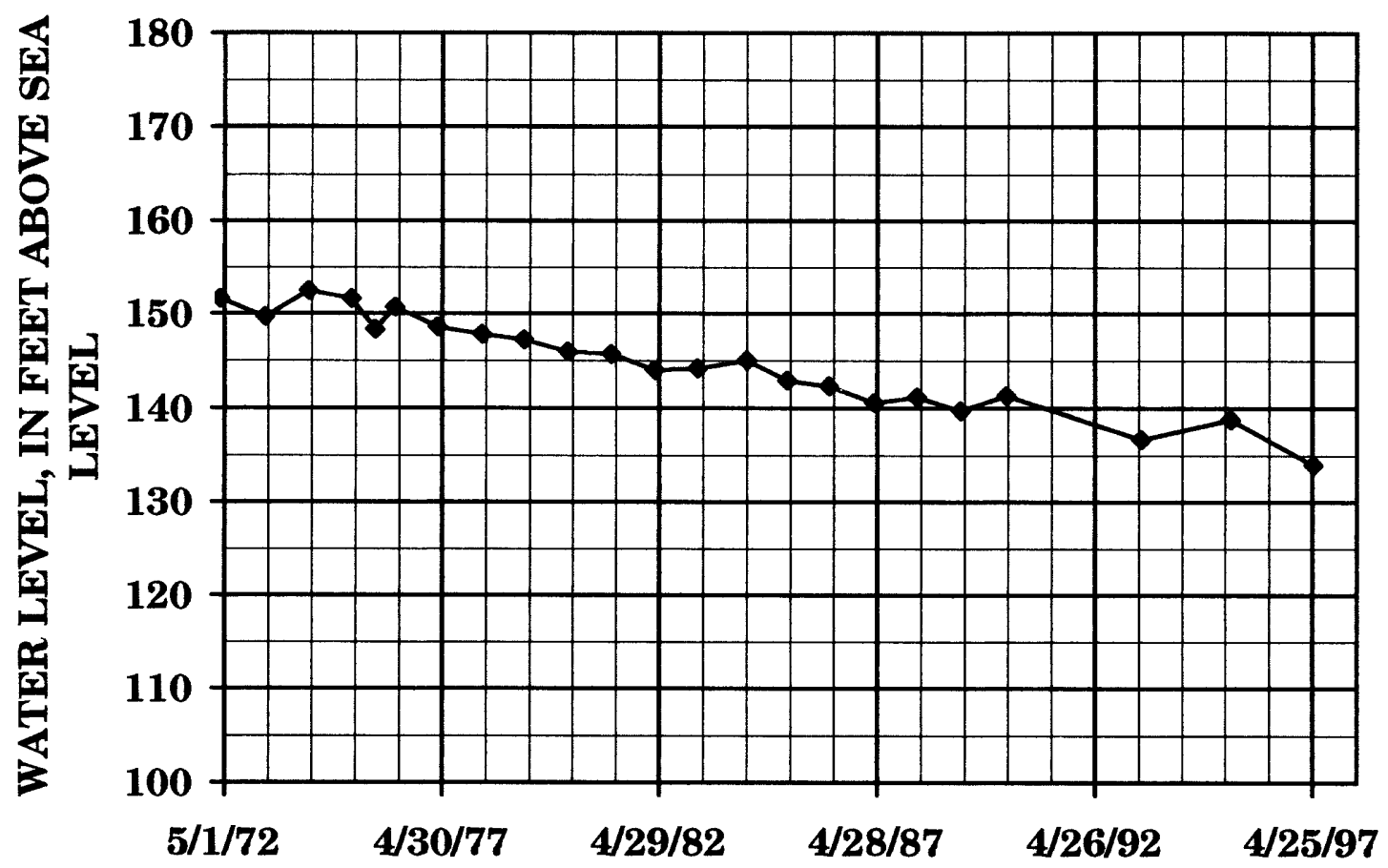

Figure 2. Water-level hydrographs for selected wells completed in the Sparta and Memphis aquifers (page 3 of 6 ). 


\section{G. POINSETT COUNTY 10N01E15DBB1}
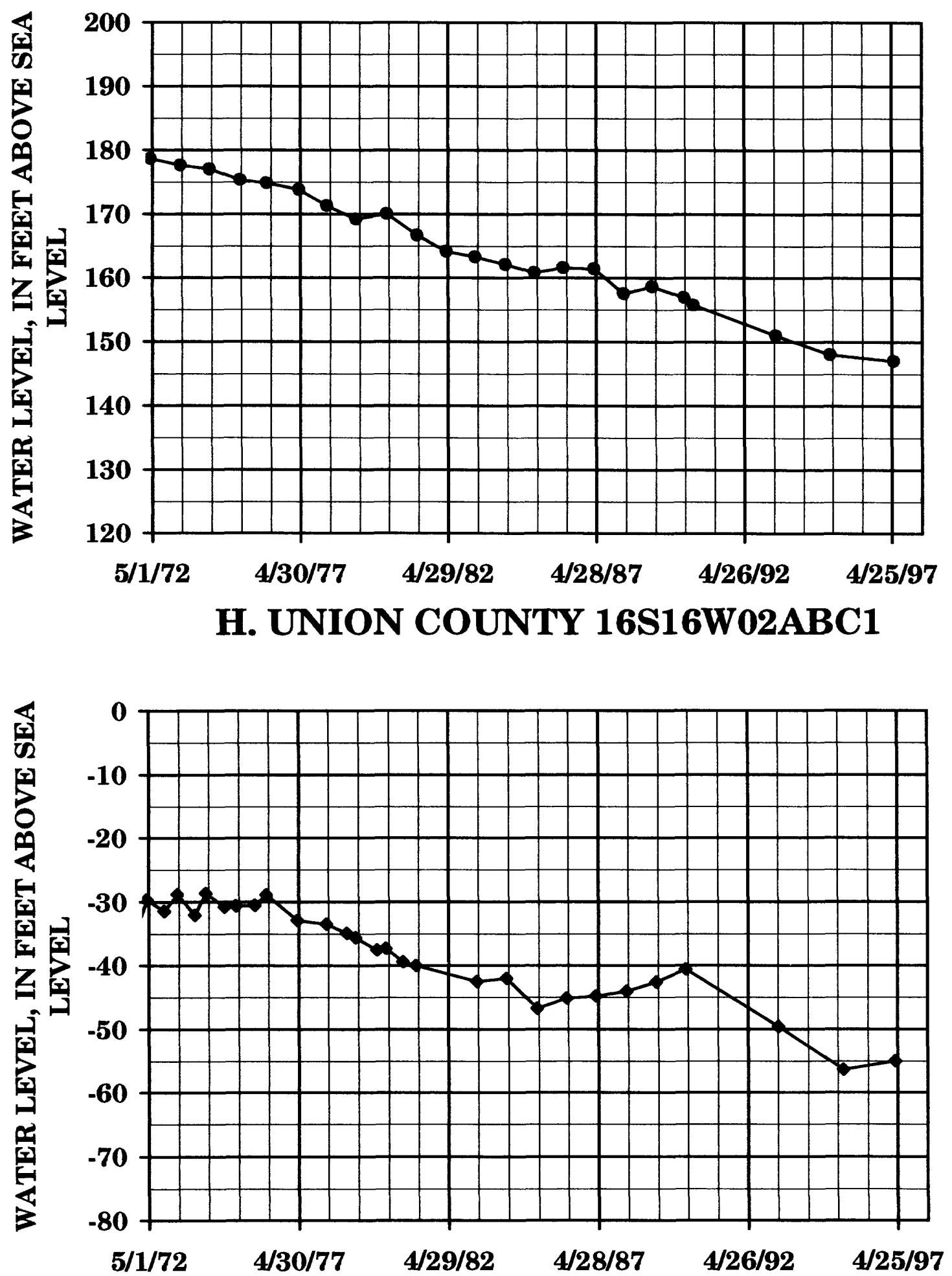

Figure 2. Water-level hydrographs for selected wells completed in the Sparta and Memphis aquifers (page 4 of 6). 


\section{CLAIBORNE PARISH Cl-149}

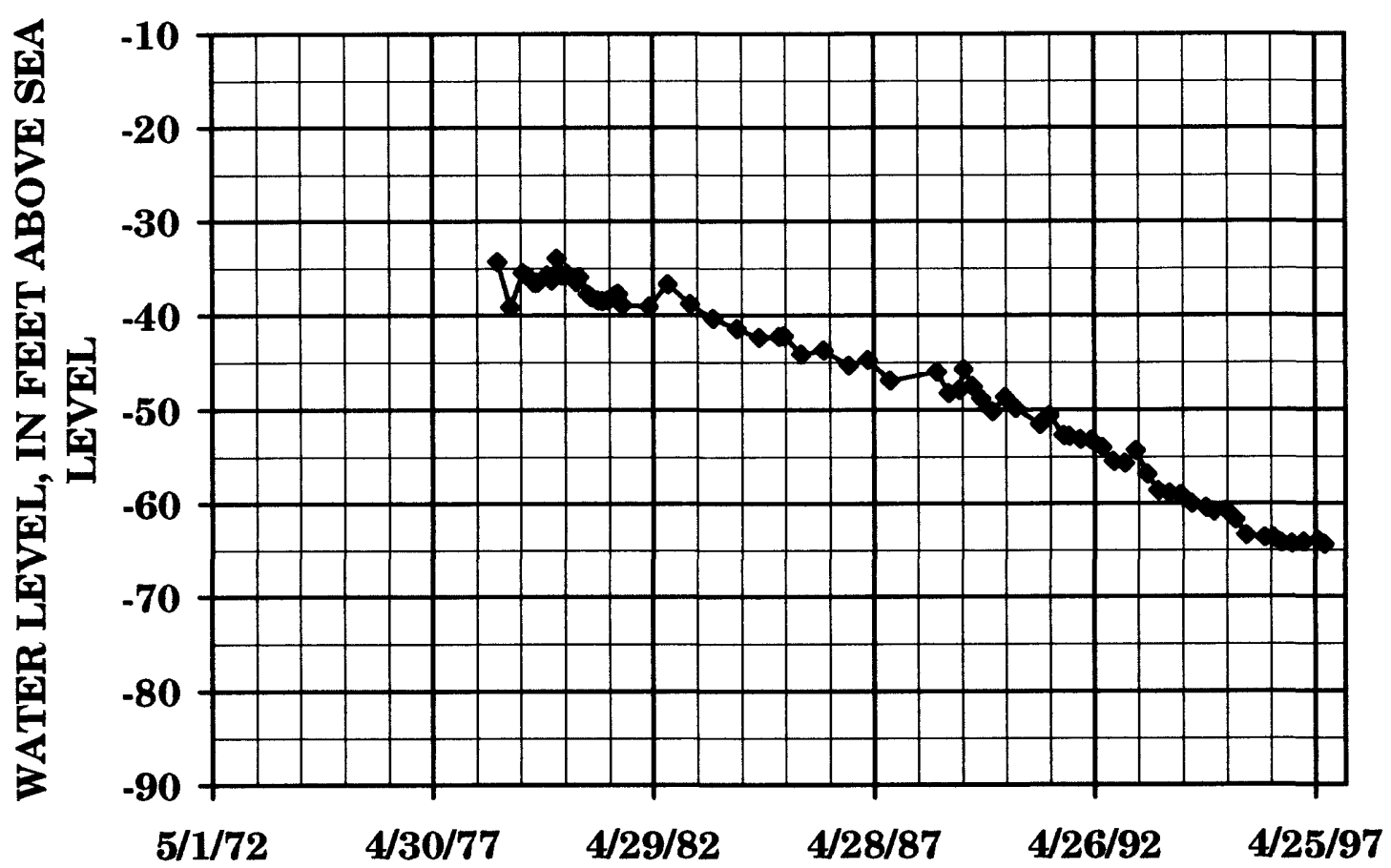

\section{J. LINCOLN PARISH L-26}

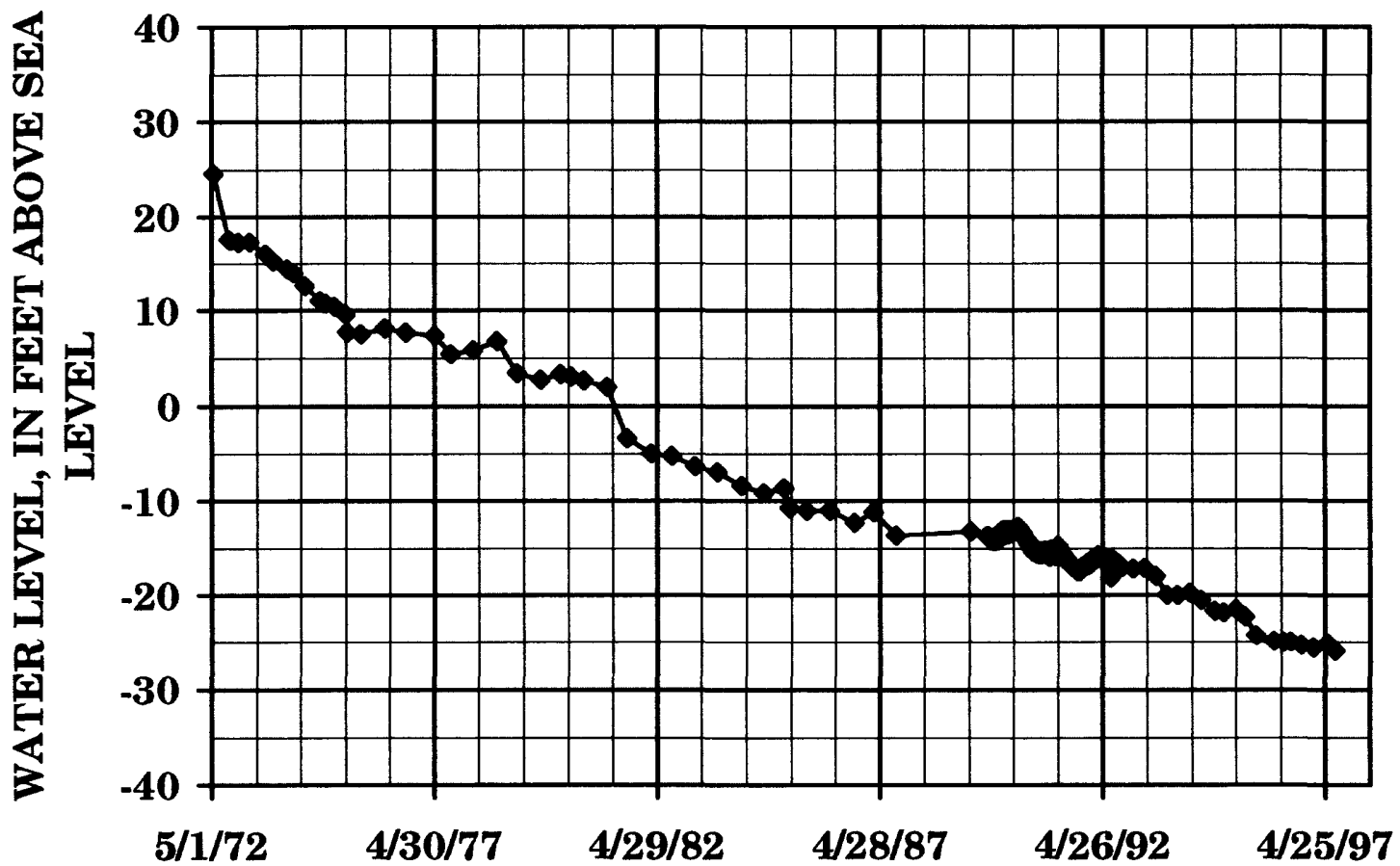

Figure 2. Water-level hydrographs for selected wells completed in the Sparta and Memphis aquifers (page 5 of 6). 


\section{K. OUACHITA PARISH Ou-444}
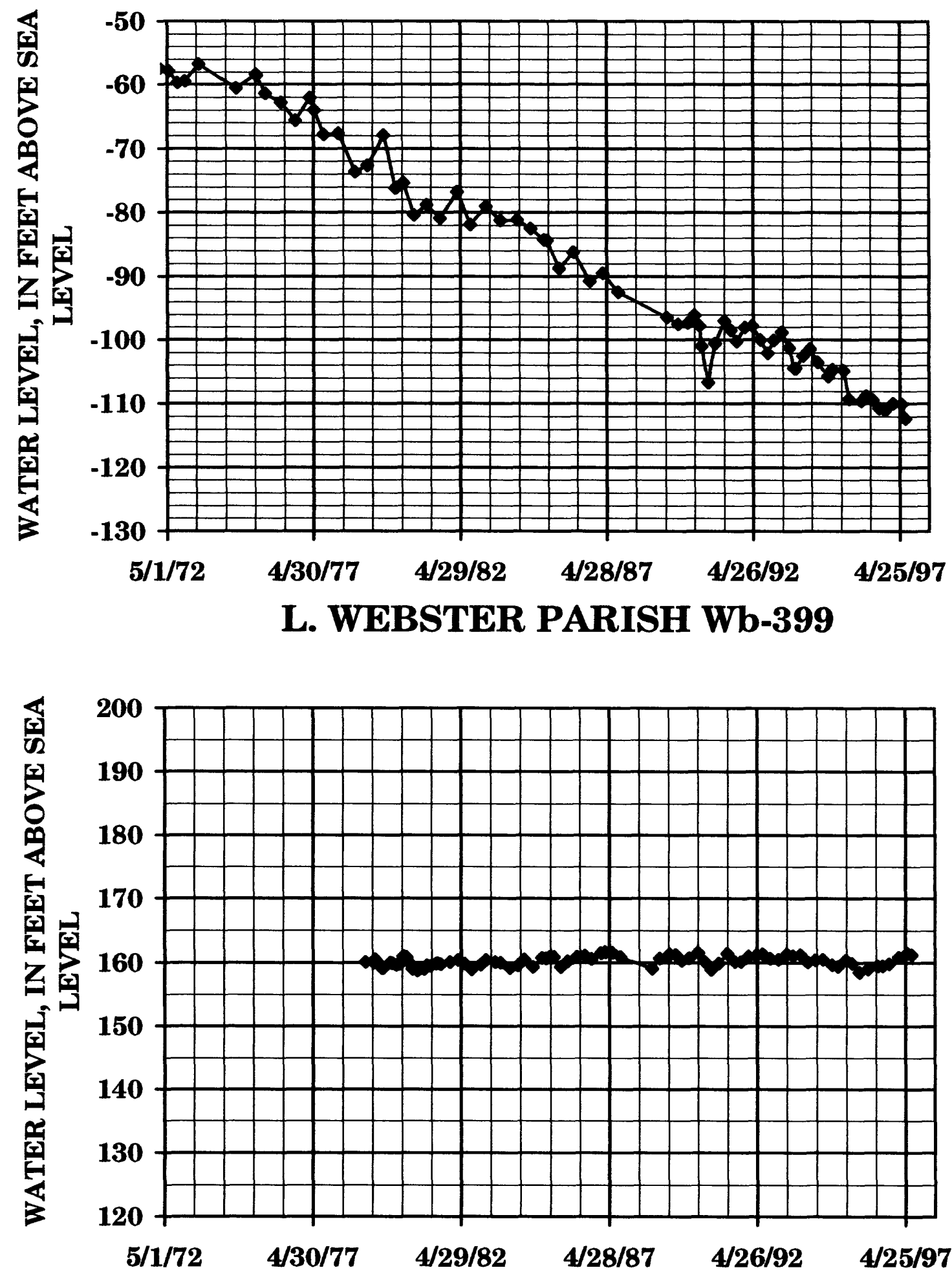

Figure 2. Water-level hydrographs for selected wells completed in the Sparta and Memphis aquifers (page 6 of 6).

18 Potentlometric Surface of the Sparta Aquifer in Eastern and South-Central Arkansas and North-Central Louisiana, and the Memphis Aquifer in East-Central Arkansas, October 1996-July 1997 


\section{SUMMARY}

During the 1997 water year, the water level in the Sparta and Memphis aquifers was measured in about 274 wells in Arkansas, and the water level in the Sparta aquifer was measured in about 55 wells in Louisiana by the U.S. Geological Survey in cooperation with the Arkansas Soil and Water Conservation Commission, the Arkansas Geological Commission, and the Louisiana Department of Transportation and Development. In Arkansas, an estimated $284 \mathrm{Mgal} / \mathrm{d}$ was withdrawn from the Sparta and Memphis aquifers in 1995, an increase of about $61 \mathrm{Mgal} / \mathrm{d}$ from 1990. In Louisiana, an estimated $71 \mathrm{Mgal} / \mathrm{d}$ was withdrawn from the Sparta aquifer in 1995, an increase of about $7 \mathrm{Mgal} / \mathrm{d}$ from 1990.

The general direction of ground-water flow in the Sparta and Memphis aquifers is from the west to the east and southeast. The regional direction of groundwater flow in Arkansas is from the north and west to the south and east, away from the recharge zone of the outcrop and subcrop area, except near areas affected by intense ground-water withdrawals; such areas are manifested by large cones of depression centered in Columbia, Jefferson, and Union Counties. The regional ground-water flow in the Sparta aquifer in north-central Louisiana generally is downdip in an easterly direction from the recharge zone of the outcrop and subcrop area in the west toward the Mississippi Alluvial Plain.

Major recharge areas in Arkansas exhibit potentiometric highs greater than 200 feet above sea level along the recharge zone of the outcrop and subcrop areas on the southern one-third of the western boundary and the northern portion of the study area in the State. The major recharge areas of Louisiana also exhibit potentiometric highs greater than 200 feet above sea level along the western boundary of the study area. In Arkansas, the potentiometric surface of the Sparta and Memphis aquifers exhibits cones of depression descending below sea level in the central and southern parts of the State. Comparison of potentiometric surface maps through time shows that the cones of depression in Columbia and Union Counties are coalescing at or near the Columbia and Union County line. In Louisiana, the potentiometric surface of the Sparta aquifer contains a cone of depression descending below sea level in the north-central parts of the State.However, the general direction of ground-water movement indicates that heavy pumpage locally has altered or reversed the natural direction of flow in some areas.
Flow in these areas is toward the cones of depression at the center of pumping.

Long-term hydrographs of 10 wells indicate trends of water-level decline over a 25-year history. During the period 1972-1997, water-level declines were less than $0.8 \mathrm{ft} / \mathrm{yr}$ in Columbia and Phillips Counties, between 1.0 and $1.5 \mathrm{ft} / \mathrm{yr}$ in Arkansas, Bradley, Desha, Jefferson, Poinsett, and Union Counties, and more than $2.0 \mathrm{ft} / \mathrm{yr}$ in Lincoln and Ouachita Parishes.

\section{SELECTED REFERENCES}

Edds, Joe, and Fitzpatrick, D.J., 1989, Altitude of the potentiometric surface and changes in water levels in the Sparta-Memphis aquifer in eastern and southern Arkansas, spring 1986: U.S. Geological Survey WaterResources Investigations Report 88-4042, 1 sheet.

Fenneman, N.M., 1938, Physiography of eastern United States: New York, McGraw-Hill Book Co., Inc., 689 p.

Holland, T.W., 1993, Use of water in Arkansas, 1990: U.S. Geological Survey Open-File Report 93-48, pamphlet.

Hosman, R.L., 1982, Outcropping Tertiary units in southern Arkansas: U.S. Geological Survey Miscellaneous Investigations Series I-1405, 1 sheet.

Hosman, R.L., Long, A.T., Lambert, T.W., and others, 1968, Tertiary aquifers in the Mississippi Embayment: U.S. Geological Survey Professional Paper 448-D, 29 p.

Payne, J.N., 1968, Hydrologic significance of the lithofacies of the Sparta Sand in Arkansas, Louisiana, Mississippi, and Texas: U.S. Geological Survey Professional Paper 569-A, $17 \mathrm{p}$.

Petersen, J.C., Broom, M.E., and Bush, W.V., 1985, Geohydrologic units of the Gulf Coastal Plain in Arkansas: U.S. Geological Survey Water-Resources Investigations Report 85-4116, 20 p.

Ryals, G.N., 1980, Potentiometric surface maps of the Sparta Sand; northern Louisiana and southern Arkansas, 1900. 1965, 1975, and 1980: U.S. Geological Survey OpenFile Report 80-1180, 1 sheet.

Smoot, C.W., and Seanor, R.C., 1991, Louisiana groundwater map no. 3: Potentiometric surface, 1989, and water-level changes, 1980-89, of the Sparta aquifer in north-central Louisiana: U.S. Geological Survey WaterResources Investigations Report 90-4183, 2 sheets.

Snider, J.L., Calandro, A.J., and Shampine, W.J., 1972, Water resources of Union Parish, Louisiana: Department of Conservation, Louisiana Geological Survey, and Louisiana Department of Public Works Water Resources Bulletin No. 17, 68 p.

Stanton, G.P., 1997, Potentiometric surface and specific conductance of the Sparta and Memphis aquifers in eastern and south-central Arkansas, 1995: U.S. Geological Survey Water-Resources Investigations Report 97-4119, $16 \mathrm{p}$. 